\title{
Statistical Analysis of the Covariance Matrix MLE in K-Distributed Clutter
}

\author{
Frederic Pascal, Alexandre Renaux
}

\begin{abstract}
In the context of radar detection, the clutter covariance matrix estimation is an important point to design optimal detectors. While the Gaussian clutter case has been extensively studied, the new advances in radar technology show that non-Gaussian clutter models have to be considered. Among these models, the Spherically Invariant Random Vector modelling is particularly interesting since it includes the K-distributed clutter model, known to fit very well with experimental data. This is why recent results in the literature focus on this distribution. More precisely, the maximum likelihood estimator of a K-distributed clutter covariance matrix has already been derived. This paper proposes a complete statistical performance analysis of this estimator through its consistency and its unbiasedness at finite number of samples. Moreover, the closed-form expression of the true Cramér-Rao bound is derived for the K-distribution covariance matrix and the efficiency of the maximum likelihood estimator is emphasized by simulations.
\end{abstract}

Key words: Covariance matrix estimation, Cramér-Rao bound, maximum likelihood, K-distribution, spherically invariant random vector.

\section{Notations}

The notational convention adopted is as follows: italic indicates a scalar quantity, as in $A$; lower case boldface indicates a vector quantity, as in a; upper case boldface indicates a matrix quantity, as in $\mathbf{A}$. $\mathcal{R} e\{A\}$ and $\mathcal{I} m\{A\}$ are the real and the imaginary parts of $A$, respectively. The complex conjugation, the matrix transpose operator and the conjugate transpose operator are indicated by ${ }^{*},{ }^{T}$, and ${ }^{H}$, respectively. The $j^{t h}$ element of a vector a is denoted $a^{(j)}$. The $n^{\text {th }}$ row and $m^{\text {th }}$ column element of the matrix $\mathbf{A}$ will be denoted by $A_{n, m} \cdot|\mathbf{A}|$ and $\operatorname{Tr}(\mathbf{A})$ are the determinant and the trace of the matrix $\mathbf{A}$, respectively. $\otimes$ denotes the Kronecker product. $\|$.$\| denotes any matrix norm. The operator$ $\operatorname{vec}(\mathbf{A})$ stacks the columns of the matrix $\mathbf{A}$ one under another into a single column vector. The operator $\operatorname{vech}(\mathbf{A})$, where $\mathbf{A}$ is a symmetric matrix, does the same things as $\operatorname{vec}(\mathbf{A})$ with the upper triangular portion excluded. The 
operator $\operatorname{veck}(\mathbf{A})$ of a skew-symmetric matrix (i.e. $\mathbf{A}^{T}=-\mathbf{A}$ ) does the same thing as $\operatorname{vech}(\mathbf{A})$ by omitting the diagonal elements. The identity matrix, with appropriate dimensions, is denoted $\mathbf{I}$ and the zero matrix is denoted $\mathbf{0}$. $E$ [.] denotes the expectation operator. $\stackrel{\text { a.s. }}{\longrightarrow}$ stands for the almost sure convergence and $\stackrel{P r}{\longrightarrow}$ stands for the convergence in probability. A zero-mean complex circular Gaussian distribution with covariance matrix $\mathbf{A}$ is denoted $\mathcal{C N}(\mathbf{0}, \mathbf{A})$. A Gamma distribution with shape parameter $k$ and scale parameter $\theta$ is denoted $\mathcal{G}(k, \theta)$. A complex $m$-variate $\mathrm{K}$-distribution with parameters $k, \theta$, and covariance matrix $\mathbf{A}$ is denoted $\mathcal{K}_{m}(k, \theta, \mathbf{A})$. A central Chi-square distribution with $k$ degrees of freedom is denoted $\chi^{2}(k)$. A uniform distribution with boundaries $a$ and $b$ is denoted $\mathcal{U}_{[a, b]}$.

\section{Introduction}

The Gaussian assumption makes sense in many applications, e.g., sources localization in passive sonar, radar detection where thermal noise and clutter are generally modelled as Gaussian processes. In these contexts, Gaussian models have been thoroughly investigated in the framework of statistical estimation and detection theory (see, e.g., [1,2] and [3]). They have led to attractive algorithms such as the stochastic maximum likelihood method $[4,5]$ or Bayesian estimators.

However, the assumption of Gaussian noise is not always valid. For instance, due to the recent evolution of radar technology, one can cite the area of Space Time Adaptive Processing-High Resolution (STAP-HR) where the resolution is such that the central limit theorem cannot be applied anymore since the number of backscatters is too small. Equivalently, it is known that reflected signals can be very impulsive when they are collected by a low grazing angle radar [6,7]. This is why, in the last decades, the radar community has been very interested in problems dealing with non-Gaussian clutter modelling (see, e.g., $[8,9,10]$ and $[11])$.

One of the most general non-Gaussian noise model is provided by Spherically Invariant Random Vectors (SIRV) which are a compound processes $[12,13,14]$. More precisely, a SIRV is the product of a Gaussian random vector (the socalled speckle) with the square root of a non-negative random scalar variable (the so-called texture). In other words, a noise modelled as a SIRV is a non-homogeneous Gaussian process with random power. Thus, these kind of processes are fully characterized by the texture and the unknown covariance matrix of the speckle. One of the major challenging difficulties in SIRV modelling is to estimate these two unknown quantities [15]. These problems have been investigated in [16] for the texture estimation while [17] and [18] have proposed different estimation procedures for the covariance matrix. Moreover, 
the knowledge of these estimates accuracy is essential in radar detection since the covariance matrix and the texture are required to design the different detection schemes.

In this context, this paper focuses on parameters estimation performance where the clutter is modelled by a K-distribution. A K-distribution is a SIRV, with a Gamma distributed texture depending on two real positive parameters $\alpha$ and $\beta$. Consequently, a K-distribution depends on $\alpha, \beta$ and on the covariance matrix M. This model choice is justified by the fact that a lot of operational data experimentations have shown the good agreement between real data and the K-distribution model (see $[7,19,20,21,22]$ and references herein).

This K-distribution model has been extensively studied in the literature. First, concerning the parameters estimation problem, [23] and [24] have estimated the Gamma distribution parameters assuming that $\mathbf{M}$ is equal to the identity matrix, [17] has proposed a recursive algorithm for the covariance matrix $\mathbf{M}$ estimation assuming $\alpha$ and $\beta$ known and [25] has used a Parameter-Expanded Expectation-Maximization (PX-EM) algorithm for the covariance matrix $\mathbf{M}$ estimation and for a parameter $\nu$ assuming $\nu=\alpha=1 / \beta$. Note also that estimation schemes in K-distribution context can be found in $[26,27]$ and references herein. Second, concerning the statistical performance of these estimators, it has been proved in [28] that the recursive scheme proposed by [17] converges and has a unique solution which is the Maximum Likelihood (ML) estimator. Consequently, this estimator has become very attractive. In order to evaluate the ultimate performance in terms of mean square error, [23] has derived the true Cramér-Rao Bound (CRB) for the parameters of the Gamma texture (namely, $\alpha$ and $\beta$ ) assuming $\mathbf{M}$ equal to the identity matrix. [29] has derived the modified CRB on the one-lag correlation coefficient of $\mathbf{M}$ where the parameters of the Gamma texture are assumed to be nuisance parameters. Concerning the covariance matrix M, a first approach for the true CRB study, which is known to be tighter than the modified one, has been proposed in [25] whatever the texture distribution. However, note that, for the particular case of a Gamma distributed texture, the analysis of [25] involves several numerical integrations and no useful information concerning the structure of the Fisher Information Matrix (FIM) is given. Finally, classical covariance matrix estimators are compared in [30] in the more general context of SIRV.

The knowledge of an accurate covariance matrix estimate is of the utmost interest in context of radar detection since this matrix is always involved in the detector expression [30]. Therefore, the goal of this contribution is twofold. First, the covariance matrix ML estimate statistical analysis is provided in terms of consistency and bias. Second, the closed-form expression of the true $\mathrm{CRB}$ for the covariance matrix $\mathbf{M}$ is given and is analyzed. Finally, through a discussion and simulation results, classical estimation procedures in Gaussian and SIRV contexts are compared. 
The paper is organized as follows. Section 2 presents the problem formulation while Sections 3 and 4 contain the main results of this paper: the ML estimate statistical performance in terms of consistency and bias and the derivation of the true CRB. Finally, Section 5 gives simulations which validate theoretical results.

\section{Problem Formulation}

In radar detection, the basic problem consists in detecting if a known signal corrupted by an additive clutter is present or not. In order to estimate the clutter parameters before detection, it is generally assumed that $K$ signalfree independent measurements, traditionally called the secondary data $\mathbf{c}_{k}$, $k=1, \ldots, K$ are available.

As stated in the introduction, one considers a clutter modelled thanks to a K-distribution denoted

$$
\mathbf{c}_{k} \sim \mathcal{K}_{m}\left(\alpha,(2 / \beta)^{2}, \mathbf{M}\right)
$$

From the SIRV definition, $\mathbf{c}_{k}$ can be written as

$$
\mathbf{c}_{k}=\sqrt{\tau_{k}} \mathbf{x}_{k}
$$

where $\tau_{k}$ is Gamma distributed with parameters $\alpha$ and $(2 / \beta)^{2}$, i.e., $\tau_{k} \sim$ $\mathcal{G}\left(\alpha,(2 / \beta)^{2}\right)$ and, where $\mathbf{x}_{k}$ is a complex circular zero-mean $m$-dimensional Gaussian vector with covariance matrix $E\left[\mathbf{x}_{k} \mathbf{x}_{k}^{H}\right]=\mathbf{M}$ independent of $\tau_{k}$. For identifiability considerations, $\mathbf{M}$ is normalized according to $\operatorname{Tr}(\mathbf{M})=m$ (see [17]). Note that the parameter $\alpha$ represents the spikiness of the clutter. Indeed, when $\alpha$ is high the clutter tends to be Gaussian and, when $\alpha$ is small, the tail of the clutter becomes heavy.

The Probability Density Function (PDF) of a random variable $\tau_{k}$ distributed according to $\mathcal{G}\left(\alpha,(2 / \beta)^{2}\right)$ is given by

$$
p\left(\tau_{k}\right)=\left(\frac{\beta^{2}}{4}\right)^{\alpha} \frac{\tau_{k}^{\alpha-1}}{\Gamma(\alpha)} \exp \left(-\frac{\beta^{2}}{4} \tau_{k}\right),
$$

where $\Gamma(\alpha)$ is the Gamma function defined by

$$
\Gamma(\alpha)=\int_{0}^{+\infty} x^{\alpha-1} \exp (-x) d x .
$$


From Eqn. (2), the PDF of $\mathbf{c}_{k}$ can be written

$$
p\left(\mathbf{c}_{k} ; \mathbf{M}, \alpha, \beta\right)=\int_{0}^{+\infty} \frac{1}{\tau_{k}^{m} \pi^{m}|\mathbf{M}|} \exp \left(-\frac{\mathbf{c}_{k}^{H} \mathbf{M}^{-1} \mathbf{c}_{k}}{\tau_{k}}\right) p\left(\tau_{k}\right) d \tau_{k},
$$

which is equal to

$$
p\left(\mathbf{c}_{k} ; \mathbf{M}, \alpha, \beta\right)=\frac{\beta^{\alpha+m}\left(\mathbf{c}_{k}^{H} \mathbf{M}^{-1} \mathbf{c}_{k}\right)^{\frac{\alpha-m}{2}}}{2^{\alpha+m-1} \pi^{m}|\mathbf{M}| \Gamma(\alpha)} K_{m-\alpha}\left(\beta \sqrt{\mathbf{c}_{k}^{H} \mathbf{M}^{-1} \mathbf{c}_{k}}\right),
$$

where $K_{\nu}($.$) is the modified Bessel function of the second kind of order \nu$ [31].

Gini et. al. have derived the ML estimator as the solution of the following equation [17]:

$$
\widehat{\mathbf{M}}_{M L}=\frac{1}{K} \sum_{k=1}^{K} c_{m}\left(\mathbf{c}_{k}^{H} \widehat{\mathbf{M}}_{M L}^{-1} \mathbf{c}_{k}\right) \mathbf{c}_{k} \mathbf{c}_{k}^{H},
$$

where the function $c_{m}(q)$ is defined as

$$
c_{m}(q)=\frac{\beta}{2 \sqrt{q}} \frac{K_{\alpha-m-1}(\beta \sqrt{q})}{K_{\alpha-m}(\beta \sqrt{q})} .
$$

Note that the ML estimate $\widehat{\mathbf{M}}_{M L}$ has to be normalized as $\mathbf{M}: \operatorname{Tr}\left(\widehat{\mathbf{M}}_{M L}\right)=m$. Finally, it has been shown in [28] that the solution of Eqn. (7) exists and is unique for the aforementioned normalization.

\section{Statistical Analysis of $\widehat{\mathbf{M}}_{M L}$}

This section is devoted to the statistical analysis of $\widehat{\mathbf{M}}_{M L}$ in terms of consistency and bias.

\subsection{Consistency}

An estimator $\widehat{\mathbf{M}}$ of $\mathbf{M}$ is said to be consistent if

$$
\|\widehat{\mathbf{M}}-\mathbf{M}\| \underset{K \rightarrow+\infty}{\stackrel{P r}{\longrightarrow}} 0,
$$

where $K$ is the number of secondary data $\mathbf{c}_{k}$ 's used to estimate $\mathbf{M}$.

Theorem 3.1 ( $\widehat{\mathrm{M}}_{M L}$ consistency)

$\widehat{\mathbf{M}}_{M L}$ is a consistent estimate of $\mathbf{M}$. 
Proof. In the sequel, $\widehat{\mathbf{M}}_{M L}$ will be denoted $\widehat{\mathbf{M}}(K)$ to show explicitly the dependence between $\widehat{\mathbf{M}}_{M L}$ and the number $K$ of $\mathbf{x}_{k}^{\prime}$ s. Let us define the function $f_{K, \mathbf{M}}$ such that

$$
f_{K, \mathbf{M}}:\left\{\begin{array}{l}
\mathcal{D} \longrightarrow \mathcal{D} \\
\mathbf{A} \longrightarrow \frac{1}{K} \sum_{k=1}^{K} c_{m}\left(\mathbf{c}_{k}^{H} \mathbf{A}^{-1} \mathbf{c}_{k}\right) \mathbf{c}_{k} \mathbf{c}_{k}^{H}
\end{array}\right.
$$

where $c_{m}($.$) is defined by Eqn. (8), where \mathcal{D}=\left\{\mathbf{A} \in M_{m}(\mathbb{C}) \mid \mathbf{A}^{H}=\mathbf{A}, \mathbf{A}\right.$ positive definite matrix $\}$ with $M_{m}(\mathbb{C})=\{m \times m$ matrices with elements in $\mathbb{C}\}$, and where $\mathbb{C}$ is the set of complex scalar. As $\widehat{\mathbf{M}}(K)$ is a fixed point of function $f_{K, \mathbf{M}}$, it is the unique zero, which respects the constraint $\operatorname{Tr}(\widehat{\mathbf{M}}(K))=m$, of the following function $g_{K}$

$$
g_{K}:\left\{\begin{array}{l}
\mathcal{D} \longrightarrow \mathcal{D} \\
\mathbf{A} \longrightarrow g_{K}(\mathbf{A})=\mathbf{A}-f_{K, \mathbf{M}}(\mathbf{A}) .
\end{array}\right.
$$

To prove the consistency of $\widehat{\mathbf{M}}(K)$, Theorem 5.9 pp. 46 of [32] will be used. First, the Strong Law of Large Numbers (SLLN) gives

$$
\forall \mathbf{A} \in \mathcal{D}, g_{K}(\mathbf{A}) \underset{K \rightarrow+\infty}{\stackrel{a . s .}{\longrightarrow}} g(\mathbf{A})
$$

where

$$
\forall \mathbf{A} \in \mathcal{D}, g(\mathbf{A})=\mathbf{A}-E\left[c_{m}\left(\mathbf{c}^{H} \mathbf{A}^{-1} \mathbf{c}\right) \mathbf{c} \mathbf{c}^{H}\right],
$$

for $\mathbf{c} \sim \mathcal{K}_{m}\left(\alpha,(2 / \beta)^{2}, \mathbf{M}\right)$.

Let us now apply the change of variable $\mathbf{y}=\mathbf{A}^{-1 / 2} \mathbf{c}$. We obtain

$$
\mathbf{y} \sim \mathcal{K}_{m}\left(\alpha,(2 / \beta)^{2}, \mathbf{A}^{-1 / 2} \mathbf{M A}^{-1 / 2}\right)
$$

and

$$
\forall \mathbf{A} \in \mathcal{D}, g(\mathbf{A})=\mathbf{A}^{1 / 2}\left(\mathbf{I}-E\left[c_{m}\left(\mathbf{y}^{H} \mathbf{y}\right) \mathbf{y} \mathbf{y}^{H}\right]\right) \mathbf{A}^{1 / 2}
$$

and

$$
\forall \mathbf{A} \in \mathcal{D}, g_{K}(\mathbf{A})=\mathbf{A}^{1 / 2}\left(\mathbf{I}-\frac{1}{K} \sum_{k=1}^{K} c_{m}\left(\mathbf{y}_{k}^{H} \mathbf{y}_{k}\right) \mathbf{y}_{k} \mathbf{y}_{k}^{H}\right) \mathbf{A}^{1 / 2}
$$

Let us verify the hypothesis of Theorem 5.9 pp. 46 of [32]. We have to prove that for every $\varepsilon>0$, 


$$
\begin{aligned}
& \left(H_{1}\right): \sup _{\mathbf{A} \in \mathcal{D}}\left\{\left\|g_{K}(\mathbf{A})-g(\mathbf{A})\right\|\right\} \frac{P r}{K \rightarrow+\infty} 0, \\
& \left(H_{2}\right): \inf _{\mathbf{A}: \| \mathbf{A}-\mathbf{M}_{\| \geq \varepsilon}\{\|g(\mathbf{A})\|\}>0=g(\mathbf{M}) .} .
\end{aligned}
$$

For every $\mathbf{A} \in \mathcal{D}$, we have

$$
\left\|g_{K}(\mathbf{A})-g(\mathbf{A})\right\|=\|\mathbf{A}\|\left\|\frac{1}{K} \sum_{k=1}^{K}\left(c_{m}\left(\mathbf{y}_{k}^{H} \mathbf{y}_{k}\right) \mathbf{y}_{k} \mathbf{y}_{k}^{H}-\mathbb{E}\left[c_{m}\left(\mathbf{y}^{H} \mathbf{y}\right) \mathbf{y} \mathbf{y}^{H}\right]\right)\right\| .
$$

Since $E\left[c_{m}\left(\mathbf{y}^{H} \mathbf{y}\right) \mathbf{y} \mathbf{y}^{H}\right]<+\infty$, one can apply the SLLN to the $K$ i.i.d. variables $c_{m}\left(\mathbf{y}_{k}^{H} \mathbf{y}_{k}\right) \mathbf{y}_{k} \mathbf{y}_{k}^{H}$, with same first order moment. This ensures $\left(H_{1}\right)$.

Moreover, the function $c_{m}\left(\mathbf{c}^{H} \mathbf{A}^{-1} \mathbf{c}\right)$ is strictly decreasing w.r.t. A. Consequently, $E\left[c_{m}\left(\mathbf{c}^{H} \mathbf{A}^{-1} \mathbf{c}\right) \mathbf{c} \mathbf{c}^{H}\right]$ too. This implies that $E\left[c_{m}\left(\mathbf{c}^{H} \mathbf{A}^{-1} \mathbf{c}\right) \mathbf{c} \mathbf{c}^{H}\right] \neq$ $\mathbf{A}$, except for $\mathbf{A}=\mathbf{M}$. This ensures $\left(H_{2}\right)$.

Finally, Theorem 5.9 pp. 46 of [32] concludes the proof and $\widehat{\mathbf{M}}_{M L} \underset{K \rightarrow+\infty}{\stackrel{P r}{\longrightarrow}} \mathbf{M}$.

\subsection{Bias}

This subsection provides an analysis of the bias $B$ defined by $B\left(\widehat{\mathbf{M}}_{M L}\right)=$ $E\left[\widehat{\mathbf{M}}_{M L}\right]-\mathbf{M}$.

\section{Theorem 3.2 (Unbiasedness of $\widehat{\mathrm{M}}_{M L}$ )}

$\widehat{\mathbf{M}}_{M L}$ is an unbiased estimate of $\mathbf{M}$ at finite distance (i.e. at finite number $K)$.

Proof. For the sake of simplicity, $\widehat{\mathbf{M}}_{M L}$ will be denoted $\widehat{\mathbf{M}}$ in this part. By applying the following change of variable, $\mathbf{y}_{k}=\mathbf{M}^{-1 / 2} \mathbf{c}_{k}$ to Eqn. (7), one has

$$
\widehat{\mathbf{M}}=\frac{1}{K} \sum_{k=1}^{K} c_{m}\left(\mathbf{y}_{k}^{H} \widehat{\mathbf{T}}^{-1} \mathbf{y}_{k}\right) \mathbf{M}^{1 / 2} \mathbf{y}_{k} \mathbf{y}_{k}^{H} \mathbf{M}^{1 / 2}
$$

where

$$
\widehat{\mathbf{T}}=\mathbf{M}^{-1 / 2} \widehat{\mathbf{M}} \mathbf{M}^{-1 / 2} .
$$

Therefore,

$$
\widehat{\mathbf{T}}=\frac{1}{K} \sum_{k=1}^{K} c_{m}\left(\mathbf{y}_{k}^{H} \widehat{\mathbf{T}}^{-1} \mathbf{y}_{k}\right) \mathbf{y}_{k} \mathbf{y}_{k}^{H}
$$


$\widehat{\mathbf{T}}$ is thus the unique estimate (see Theorem III.1 of [28]) of the identity matrix, with $\operatorname{Tr}(\widehat{\mathbf{T}})=m$. Its statistic is clearly independent of $\mathbf{M}$ since the $\mathbf{y}_{k}$ 's are i.i.d. SIRVs with a Gamma distributed texture and identity matrix for the Gaussian covariance matrix. In other words, $\mathbf{y}_{k} \sim \mathcal{K}_{m}\left(\alpha,(2 / \beta)^{2}, \mathbf{I}\right)$.

Moreover, for any unitary matrix $\mathbf{U}$,

$$
\mathbf{U} \widehat{\mathbf{T}} \mathbf{U}^{H}=\frac{1}{K} \sum_{k=1}^{K} c_{m}\left(\mathbf{z}_{k}^{H}\left(\mathbf{U} \widehat{\mathbf{T}} \mathbf{U}^{H}\right)^{-1} \mathbf{z}_{k}\right) \mathbf{z}_{k} \mathbf{z}_{k}^{H},
$$

where $\mathbf{z}_{k}=\mathbf{U} \mathbf{y}_{k}$ are also i.i.d. and distributed as $\mathcal{K}_{m}\left(\alpha,(2 / \beta)^{2}, \mathbf{I}\right)$ and $\mathbf{U} \widehat{\mathbf{T}} \mathbf{U}^{H}$ has the same distribution as $\widehat{\mathbf{T}}$. Consequently,

$$
E[\widehat{\mathbf{T}}]=\mathbf{U} E[\widehat{\mathbf{T}}] \mathbf{U}^{H} \text {, for any unitary matrix } \mathbf{U} \text {. }
$$

Since $E[\widehat{\mathbf{T}}]$ is different from $\mathbf{0}$, Lemma A.1 of [30] ensures that $E[\widehat{\mathbf{T}}]=\gamma \mathbf{I}$ for $\gamma \in \mathbb{R}$. Remind that $\widehat{\mathbf{T}}=\mathbf{M}^{-1 / 2} \widehat{\mathbf{M}} \mathbf{M}^{-1 / 2}$, then $E[\widehat{\mathbf{M}}]=\gamma \mathbf{M}$. Moreover, since $\operatorname{Tr}(\widehat{\mathbf{M}})=\operatorname{Tr}(\mathbf{M})=m$, one has

$$
m=E(\operatorname{Tr}(\widehat{\mathbf{M}}))=\operatorname{Tr}(E(\widehat{\mathbf{M}}))=\gamma \operatorname{Tr}(\mathbf{M})=\gamma m,
$$

which implies that $\gamma=1$.

In conclusion, $\widehat{\mathbf{M}}$ is an unbiased estimate of $\mathbf{M}$, for any number $K$ of secondary data.

\subsection{Comments}

Theorems 3.1 and 3.2 show the attractiveness of the estimator (7) in terms of statistical properties, i.e., consistency and unbiasedness. Note also that this estimator is robust since the unbiasedness property is at finite number of samples. In the next section, the Cramér-Rao bound is derived for the observation model (2).

\section{Cramér-Rao bound}

In this Section, the Cramér-Rao bound w.r.t. $\mathbf{M}$ is derived. The CRB gives the best variance that an unbiased estimator can achieve. The proposed bound will 
be compared to the mean square error of the previously studied ML estimator (Eqn. (7)) in the next section.

The CRB for a parameter vector $\boldsymbol{\theta}$ is given by

$$
\mathbf{C R B}_{\boldsymbol{\theta}}=\left(-E\left[\frac{\partial^{2} \ln p\left(\mathbf{c}_{1}, \ldots, \mathbf{c}_{K} ; \boldsymbol{\theta}\right)}{\partial \boldsymbol{\theta} \partial \boldsymbol{\theta}^{T}}\right]\right)^{-1},
$$

where $p\left(\mathbf{c}_{1}, \ldots, \mathbf{c}_{K} ; \boldsymbol{\theta}\right)$ is the likelihood function of the observations $\mathbf{c}_{k}, k=$ $1 \ldots K$. Concerning our model, the parameter vector is

$$
\boldsymbol{\theta}=\left(\operatorname{vech}^{T}(\mathcal{R} e\{\mathbf{M}\}) \operatorname{veck}^{T}(\mathcal{I} m\{\mathbf{M}\})\right)^{T}, \quad m^{2} \times 1
$$

With the parametrization of Eqn.(13), the structure of the CRB becomes

$$
\mathrm{CRB}_{\boldsymbol{\theta}}=\left(\begin{array}{ll}
\mathbf{F}_{1,1} & \mathbf{F}_{1,2} \\
\mathbf{F}_{2,1} & \mathbf{F}_{2,2}
\end{array}\right)^{-1}
$$

where the $\mathbf{F}_{i, j}$ 's are the elements of the FIM given by

$$
\begin{aligned}
& \mathbf{F}_{1,1}=-E\left[\frac{\partial^{2} \ln p\left(\mathbf{c}_{1}, \ldots, \mathbf{c}_{K} ; \boldsymbol{\theta}\right)}{\partial \operatorname{vech}(\mathcal{R} e\{\mathbf{M}\}) \partial \operatorname{vech}^{T}(\mathcal{R} e\{\mathbf{M}\})}\right] \frac{m(m+1)}{2} \times \frac{m(m+1)}{2}, \\
& \mathbf{F}_{1,2}= \mathbf{F}_{2,1}^{T}=-E\left[\frac{\partial^{2} \ln p\left(\mathbf{c}_{1}, \ldots, \mathbf{c}_{K} ; \boldsymbol{\theta}\right)}{\partial \operatorname{vech}(\mathcal{R} e\{\mathbf{M}\}) \partial \operatorname{veck}^{T}(\mathcal{I} m\{\mathbf{M}\})}\right], \\
& \frac{m(m+1)}{2} \times \frac{m(m-1)}{2}, \\
& \mathbf{F}_{2,2}=-E\left[\frac{\partial^{2} \ln p\left(\mathbf{c}_{1}, \ldots, \mathbf{c}_{K} ; \boldsymbol{\theta}\right)}{\partial \operatorname{veck}(\mathcal{I} m\{\mathbf{M}\}) \partial \operatorname{veck}^{T}(\mathcal{I} m\{\mathbf{M}\})}\right] \\
& \frac{m(m-1)}{2} \times \frac{m(m-1)}{2} .
\end{aligned}
$$

Since the $\mathbf{c}_{k}$ 's are i.i.d. random vectors, one have from Eqn. (6),

$$
p\left(\mathbf{c}_{1}, \ldots, \mathbf{c}_{K} ; \boldsymbol{\theta}\right)=\prod_{k=1}^{K} \frac{\beta^{\alpha+m}\left(\mathbf{c}_{k}^{H} \mathbf{M}^{-1} \mathbf{c}_{k}\right)^{\frac{\alpha-m}{2}}}{2^{\alpha+m-1} \pi^{m}|\mathbf{M}| \Gamma(\alpha)} K_{m-\alpha}\left(\beta \sqrt{\mathbf{c}_{k}^{H} \mathbf{M}^{-1} \mathbf{c}_{k}}\right)
$$

Consequently, the log-likelihood function can be written 


$$
\begin{aligned}
\ln p\left(\mathbf{c}_{1}, \ldots, \mathbf{c}_{K} \mid \boldsymbol{\theta}\right) & =K \ln \left(\frac{\beta^{\alpha+m}}{2^{\alpha+m-1} \pi^{m} \Gamma(\alpha)}\right)-K \ln (|\mathbf{M}|) \\
& +\sum_{k=1}^{K} \ln \left(\left(\mathbf{c}_{k}^{H} \mathbf{M}^{-1} \mathbf{c}_{k}\right)^{\frac{\alpha-m}{2}} K_{m-\alpha}\left(\beta \sqrt{\mathbf{c}_{k}^{H} \mathbf{M}^{-1} \mathbf{c}_{k}}\right)\right)
\end{aligned}
$$

\subsection{Result}

The next sub-sections will show that the CRB w.r.t. $\boldsymbol{\theta}$, in this context, is given by

$$
\mathbf{C R B}_{\boldsymbol{\theta}}=\frac{1}{K}\left(\begin{array}{cc}
\left(\mathbf{H}^{T} \mathbf{F H}\right)^{-1} & \mathbf{0} \\
\mathbf{0} & \left(\mathbf{K}^{T} \mathbf{F K}\right)^{-1}
\end{array}\right),
$$

where the matrices $\mathbf{H}$ and $\mathbf{K}$ are constant transformation matrices filled with ones and zeros such that $\operatorname{vec}(\mathbf{A})=\mathbf{H} \operatorname{vech}(\mathbf{A})$ and $\operatorname{vec}(\mathbf{A})=\operatorname{Kveck}(\mathbf{A})$, with A a skew-symmetric matrix, and where

$$
\begin{aligned}
& \mathbf{F}=\left(\mathbf{M}^{T} \otimes \mathbf{M}\right)^{-1}- \\
& \left(\frac{2(\alpha+1)}{m+1}-\frac{\varphi(\alpha, m)}{8}\right)\left(\mathbf{M}^{T} \otimes \mathbf{M}\right)^{-1 / 2}\left(\mathbf{I}+\operatorname{vec}(\mathbf{I}) \operatorname{vec}^{T}(\mathbf{I})\right)\left(\mathbf{M}^{T} \otimes \mathbf{M}\right)^{-1 / 2}
\end{aligned}
$$

where $\varphi(\alpha, m)$ is given by Eqn. (F.3).

\section{Remarks:}

- $\varphi(\alpha, m)$ is a constant which does not depend on $\beta$ since $\beta^{2} \tau_{k^{\prime}} \sim \mathcal{G}(\alpha, 4)$ (see Eqn. (F.3)).

- The first term of the right hand side of Eqn. (24) is the Gaussian FIM (i.e., when $\tau_{k}=1 \forall k$ ). Indeed, using Eqn. (27), (52) and (33) ${ }^{1}$, the Gaussian CRB, denoted GCRB $_{\boldsymbol{\theta}}$, is straightforwardly obtained

$$
\mathbf{G C R B}_{\boldsymbol{\theta}}=\frac{1}{K}\left(\begin{array}{cc}
\left(\mathbf{H}^{T}\left(\mathbf{M}^{T} \otimes \mathbf{M}\right)^{-1} \mathbf{H}\right)^{-1} & \mathbf{0} \\
\mathbf{0} & \left(\mathbf{K}^{T}\left(\mathbf{M}^{T} \otimes \mathbf{M}\right)^{-1} \mathbf{K}\right)^{-1}
\end{array}\right)
$$

By identification with Eqn.(24), it means that $\lim _{\alpha \rightarrow \infty}\left(\frac{2(\alpha+1)}{m+1}-\frac{\gamma}{8}\right)=0$. Consequently, due to the structure of $\mathbf{I}+\operatorname{vec}(\mathbf{I}) \operatorname{vec}^{T}(\mathbf{I})$, the FIM for K-distributed observations is given by the Gaussian FIM minus a sparse matrix depending on $\alpha, \mathbf{M}$ and $m$.

$\overline{1 \text { With }} \beta^{2}=1, z=\mathbf{c}_{k}^{H} \mathbf{M}^{-1} \mathbf{c}_{k}$ which is the term to be derived w.r.t. $\boldsymbol{\theta}$ in the exponential term of the multivariate Gaussian distribution. 


\subsection{Outline of the proof}

To make the reading easier, only the outline of the CRB derivation is given below. All the details are reported into the different appendices.

\subsubsection{Analysis of $\mathbf{F}_{1,1}$}

With Eqn. (22) one has

$$
\begin{gathered}
\frac{\partial^{2} \ln p\left(\mathbf{c}_{1}, \ldots, \mathbf{c}_{K} ; \boldsymbol{\theta}\right)}{\partial \operatorname{vech}(\mathcal{R} e\{\mathbf{M}\}) \partial \operatorname{vech}^{T}(\mathcal{R} e\{\mathbf{M}\})}=-K \frac{\partial^{2} \ln (|\mathbf{M}|)}{\partial \operatorname{vech}(\mathcal{R} e\{\mathbf{M}\}) \partial \operatorname{vech}^{T}(\mathcal{R} e\{\mathbf{M}\})} \\
+\sum_{k=1}^{K} \frac{\partial^{2} \ln \left(\left(\mathbf{c}_{k}^{H} \mathbf{M}^{-1} \mathbf{c}_{k}\right)^{\frac{\alpha-m}{2}} K_{m-\alpha}\left(\beta \sqrt{\mathbf{c}_{k}^{H} \mathbf{M}^{-1} \mathbf{c}_{k}}\right)\right)}{\partial \operatorname{vech}(\mathcal{R} e\{\mathbf{M}\}) \partial \operatorname{vech}^{T}(\mathcal{R} e\{\mathbf{M}\})}
\end{gathered}
$$

The first part of the right hand-side of Eqn. (26) is given by

$$
-K \frac{\partial^{2} \ln (|\mathbf{M}|)}{\partial \operatorname{vech}(\mathcal{R} e\{\mathbf{M}\}) \partial \operatorname{vech}^{T}(\mathcal{R} e\{\mathbf{M}\})}=K \mathbf{H}^{T}\left(\mathbf{M}^{T} \otimes \mathbf{M}\right)^{-1} \mathbf{H}
$$

thanks to Appendix A, Eqn. (A.3), and thanks to the fact that

$$
\frac{\partial \operatorname{veck}^{T}(\mathcal{I} m\{\mathbf{M}\})}{\partial \operatorname{vech}(\mathcal{R} e\{\mathbf{M}\})}=\mathbf{0}
$$

and

$$
\frac{\partial \operatorname{vech}^{T}(\mathcal{R} e\{\mathbf{M}\})}{\partial \operatorname{vech}(\mathcal{R} e\{\mathbf{M}\})}=\mathbf{I}
$$

Through the remain of the paper, let us set $z=\beta^{2} \mathbf{c}_{k}^{H} \mathbf{M}^{-1} \mathbf{c}_{k}$ and $f(z)=$ $\ln \left(\left(\frac{z}{\beta^{2}}\right)^{\frac{\alpha-m}{2}} K_{m-\alpha}(\sqrt{z})\right)$. The second term (inside the sum) of the right-hand side of Eqn. (26) is given by

$$
\begin{gathered}
\frac{\partial^{2} \ln \left(\left(\mathbf{c}_{k}^{H} \mathbf{M}^{-1} \mathbf{c}_{k}\right)^{\frac{\alpha-m}{2}} K_{m-\alpha}\left(\beta \sqrt{\mathbf{c}_{k}^{H} \mathbf{M}^{-1} \mathbf{c}_{k}}\right)\right)}{\partial \operatorname{vech}(\mathcal{R} e\{\mathbf{M}\}) \partial \operatorname{vech}^{T}(\mathcal{R} e\{\mathbf{M}\})} \\
=\frac{\partial^{2} f(z)}{\partial \operatorname{vech}(\mathcal{R} e\{\mathbf{M}\}) \partial \operatorname{vech}^{T}(\mathcal{R e}\{\mathbf{M}\})},
\end{gathered}
$$

Note that 


$$
\begin{aligned}
\partial^{2} f(z) & =\frac{\partial}{\partial z}\left(\frac{\partial f(z)}{\partial z} \partial z\right) \partial z \\
& =\frac{\partial^{2} f(z)}{\partial z^{2}} \partial z \partial z+\frac{\partial f(z)}{\partial z} \partial^{2} z
\end{aligned}
$$

with (details are given in Appendix B)

$$
\begin{aligned}
\partial z= & -\beta^{2} \partial \operatorname{vech}^{T}(\mathcal{R} e\{\mathbf{M}\}) \mathbf{H}^{T}\left(\mathbf{M}^{T} \otimes \mathbf{M}\right)^{-1} \operatorname{vec}\left(\mathbf{c}_{k} \mathbf{c}_{k}^{H}\right) \\
& +i \beta^{2} \partial \operatorname{veck}^{T}(\mathcal{I} m\{\mathbf{M}\}) \mathbf{K}^{T}\left(\mathbf{M}^{T} \otimes \mathbf{M}\right)^{-1} \operatorname{vec}\left(\mathbf{c}_{k} \mathbf{c}_{k}^{H}\right),
\end{aligned}
$$

and

$$
\begin{aligned}
& \partial^{2} z= \\
& 2 \beta^{2} \partial \operatorname{vech}^{T}(\mathcal{R} e\{\mathbf{M}\}) \mathbf{H}^{T}\left(\left(\mathbf{M}^{-T} \mathbf{c}_{k}^{*} \mathbf{c}_{k}^{T} \mathbf{M}^{-T}\right) \otimes \mathbf{M}^{-1}\right) \mathbf{H} \partial \operatorname{vech}(\mathcal{R} e\{\mathbf{M}\}) \\
& +2 \beta^{2} \partial \operatorname{veck}^{T}(\mathcal{I} m\{\mathbf{M}\}) \mathbf{K}^{T}\left(\left(\mathbf{M}^{-T} \mathbf{c}_{k}^{*} \mathbf{c}_{k}^{T} \mathbf{M}^{-T}\right) \otimes \mathbf{M}^{-1}\right) \mathbf{K} \partial \operatorname{veck}(\mathcal{I} m\{\mathbf{M}\}),
\end{aligned}
$$

and (details are given in Appendix C)

$$
d_{1}(z)=\frac{\partial f(z)}{\partial z}=-\frac{1}{2 \sqrt{z}} \frac{K_{m-\alpha+1}(\sqrt{z})}{K_{m-\alpha}(\sqrt{z})}
$$

and

$$
d_{2}(z)=\frac{\partial^{2} f(z)}{\partial z^{2}}=\frac{1}{4 z}\left(1+\frac{K_{m-\alpha+1}(\sqrt{z})}{K_{m-\alpha}(\sqrt{z})}\left(\frac{2}{\sqrt{z}}-\frac{K_{m-\alpha-1}(\sqrt{z})}{K_{m-\alpha}(\sqrt{z})}\right)\right) .
$$

Consequently, the structure of Eqn. (31) becomes

$$
\begin{aligned}
\partial^{2} f(z)= & 2 \beta^{2} d_{1}(z) \partial \operatorname{vech}^{T}(\mathcal{R} e\{\mathbf{M}\}) \mathbf{H}^{T} \mathbf{P}_{1} \mathbf{H} \partial \operatorname{vech}(\mathcal{R} e\{\mathbf{M}\}) \\
& +2 \beta^{2} d_{1}(z) \partial \operatorname{veck}^{T}(\mathcal{I} m\{\mathbf{M}\}) \mathbf{K}^{T} \mathbf{P}_{1} \mathbf{K} \partial \operatorname{veck}(\mathcal{I} m\{\mathbf{M}\}) \\
& +\beta^{4} d_{2}(z) \partial \operatorname{vech}^{T}(\mathcal{R} e\{\mathbf{M}\}) \mathbf{H}^{T} \mathbf{P}_{2} \mathbf{H} \partial \operatorname{vech}(\mathcal{R} e\{\mathbf{M}\}) \\
& +\beta^{4} d_{2}(z) \partial \operatorname{veck}^{T}(\mathcal{I} m\{\mathbf{M}\}) \mathbf{K}^{T} \mathbf{P}_{2} \mathbf{K} \partial \operatorname{veck}(\mathcal{I} m\{\mathbf{M}\}),
\end{aligned}
$$

where

$$
\begin{aligned}
& \mathbf{P}_{1}=\left(\mathbf{M}^{-T} \mathbf{c}_{k}^{*} \mathbf{c}_{k}^{T} \mathbf{M}^{-T}\right) \otimes \mathbf{M}^{-1} \\
& \mathbf{P}_{2}=\left(\mathbf{M}^{T} \otimes \mathbf{M}\right)^{-1} \operatorname{vec}\left(\mathbf{c}_{k} \mathbf{c}_{k}^{H}\right) \operatorname{vec}^{H}\left(\mathbf{c}_{k} \mathbf{c}_{k}^{H}\right)\left(\mathbf{M}^{T} \otimes \mathbf{M}\right)^{-1} .
\end{aligned}
$$

Therefore, Eqn. (30) is given by 


$$
\frac{\partial^{2} f(z)}{\partial \operatorname{vech}(\mathcal{R} e\{\mathbf{M}\}) \partial \operatorname{vech}^{T}(\mathcal{R} e\{\mathbf{M}\})}=2 \beta^{2} d_{1}(z) \mathbf{H}^{T} \mathbf{P}_{1} \mathbf{H}+\beta^{4} d_{2}(z) \mathbf{H}^{T} \mathbf{P}_{2} \mathbf{H}
$$

due to Eqn. (28) and Eqn. (29).

Using Eqn. (26), Eqn. (27), and Eqn. (39), $\mathbf{F}_{1,1}$ is given by

$$
\mathbf{F}_{1,1}=-K \mathbf{H}^{T}\left(\left(\mathbf{M}^{T} \otimes \mathbf{M}\right)^{-1}+2 \beta^{2} E\left[d_{1}(z) \mathbf{P}_{1}\right]+\beta^{4} E\left[d_{2}(z) \mathbf{P}_{2}\right]\right) \mathbf{H},
$$

where $d_{1}(z)$ and $d_{2}(z)$ are defined by Eqn. (34) and Eqn. (35), respectively.

The two expectation operators involved in the previous equation can be detailed as follows:

$$
E\left[d_{1}(z) \mathbf{P}_{1}\right]=-\frac{1}{2}\left(\mathbf{M}^{-T} \mathbf{\Gamma} \mathbf{M}^{-T}\right) \otimes \mathbf{M}^{-1},
$$

and

$$
E\left[d_{2}(z) \mathbf{P}_{2}\right]=\frac{1}{4}\left(\mathbf{M}^{T} \otimes \mathbf{M}\right)^{-1}(\mathbf{\Psi}+\mathbf{\Xi}-\mathbf{\Upsilon})\left(\mathbf{M}^{T} \otimes \mathbf{M}\right)^{-1},
$$

where

$$
\left\{\begin{array}{l}
\boldsymbol{\Gamma}=E\left[\frac{1}{\sqrt{z}} \frac{K_{m-\alpha+1}(\sqrt{z})}{K_{m-\alpha}(\sqrt{z})} \mathbf{c}_{k}^{*} \mathbf{c}_{k}^{T}\right], \\
\boldsymbol{\Psi}=E\left[\frac{1}{z} \operatorname{vec}\left(\mathbf{c}_{k} \mathbf{c}_{k}^{H}\right) \operatorname{vec}^{H}\left(\mathbf{c}_{k} \mathbf{c}_{k}^{H}\right)\right], \\
\boldsymbol{\Xi}=E\left[\frac{2}{z^{\frac{3}{2}}} \frac{K_{m-\alpha+1}(\sqrt{z})}{K_{m-\alpha}(\sqrt{z})} \operatorname{vec}\left(\mathbf{c}_{k} \mathbf{c}_{k}^{H}\right) \operatorname{vec}^{H}\left(\mathbf{c}_{k} \mathbf{c}_{k}^{H}\right)\right], \\
\boldsymbol{\Upsilon}=E\left[\frac{1}{z} \frac{K_{m-\alpha-1}(\sqrt{z}) K_{m-\alpha+1}(\sqrt{z})}{K_{m-\alpha}^{2}(\sqrt{z})} \operatorname{vec}\left(\mathbf{c}_{k} \mathbf{c}_{k}^{H}\right) \operatorname{vec}^{H}\left(\mathbf{c}_{k} \mathbf{c}_{k}^{H}\right)\right] .
\end{array}\right.
$$

After some calculus detailed in Appendix D, one finds

$$
\boldsymbol{\Gamma}=\frac{2}{\beta^{2}} \mathbf{M}^{T} .
$$

Concerning $\Psi$, one has

$$
\boldsymbol{\Psi}=\frac{1}{\beta^{2}} E\left[\frac{\operatorname{vec}\left(\mathbf{c}_{k} \mathbf{c}_{k}^{H}\right) \operatorname{vec}^{H}\left(\mathbf{c}_{k} \mathbf{c}_{k}^{H}\right)}{\mathbf{c}_{k}^{H} \mathbf{M}^{-1} \mathbf{c}_{k}}\right],
$$

where the expectation is taken under a complex K-distribution $\mathcal{K}_{m}\left(\alpha,(2 / \beta)^{2}, \mathbf{M}\right)$. 
Concerning $\boldsymbol{\Xi}$, one has

$$
\boldsymbol{\Xi}=\frac{1}{(\alpha-1) \beta^{2}} E\left[\frac{\operatorname{vec}\left(\mathbf{c}_{k} \mathbf{c}_{k}^{H}\right) \operatorname{vec}^{H}\left(\mathbf{c}_{k} \mathbf{c}_{k}^{H}\right)}{\mathbf{c}_{k}^{H} \mathbf{M}^{-1} \mathbf{c}_{k}}\right]
$$

where the expectation is taken under a complex K-distribution $\mathcal{K}_{m}\left(\alpha-1,(2 / \beta)^{2}, \mathbf{M}\right)$. Concerning $\Upsilon$, one has

$$
\mathbf{\Upsilon}=\frac{1}{\beta^{2}} E\left[\frac{K_{m-\alpha-1}(\sqrt{z}) K_{m-\alpha+1}(\sqrt{z})}{K_{m-\alpha}^{2}(\sqrt{z})} \frac{\operatorname{vec}\left(\mathbf{c}_{k} \mathbf{c}_{k}^{H}\right) \operatorname{vec}^{H}\left(\mathbf{c}_{k} \mathbf{c}_{k}^{H}\right)}{\mathbf{c}_{k}^{H} \mathbf{M}^{-1} \mathbf{c}_{k}}\right]
$$

where the expectation is taken under a complex $\mathrm{K}$-distribution $\mathcal{K}_{m}\left(\alpha,(2 / \beta)^{2}, \mathbf{M}\right)$.

The closed-form expression of $E\left[\frac{\operatorname{vec}\left(\mathbf{c}_{k} \mathbf{c}_{k}^{H}\right) \operatorname{vec}^{H}\left(\mathbf{c}_{k} \mathbf{c}_{k}^{H}\right)}{\mathbf{c}_{k}^{H} \mathbf{M}^{-1} \mathbf{c}_{k}}\right]$ under a complex Kdistribution is given in Appendix E. One finds

$$
\boldsymbol{\Psi}=\frac{8}{\beta^{4}} \frac{\alpha}{m+1}\left(\mathbf{M}^{T / 2} \otimes \mathbf{M}^{1 / 2}\right)\left(\mathbf{I}+\operatorname{vec}(\mathbf{I}) \operatorname{vec}^{T}(\mathbf{I})\right)\left(\mathbf{M}^{T / 2} \otimes \mathbf{M}^{1 / 2}\right),
$$

and

$$
\boldsymbol{\Xi}=\frac{8}{\beta^{4}} \frac{1}{m+1}\left(\mathbf{M}^{T / 2} \otimes \mathbf{M}^{1 / 2}\right)\left(\mathbf{I}+\operatorname{vec}(\mathbf{I}) \operatorname{vec}^{T}(\mathbf{I})\right)\left(\mathbf{M}^{T / 2} \otimes \mathbf{M}^{1 / 2}\right) .
$$

The structure of $\Upsilon$ is analyzed in Appendix F.

Consequently, Eqn. (40) is reduced to

$$
\begin{aligned}
& \mathbf{F}_{1,1}=K \mathbf{H}^{T}\left(\left(\mathbf{M}^{T} \otimes \mathbf{M}\right)^{-1}-\right. \\
& \left.\left(\frac{2(\alpha+1)}{m+1}-\frac{\varphi(\alpha, m)}{8}\right)\left(\mathbf{M}^{T} \otimes \mathbf{M}\right)^{-1 / 2}\left(\mathbf{I}+\operatorname{vec}(\mathbf{I}) \operatorname{vec}^{T}(\mathbf{I})\right)\left(\mathbf{M}^{T} \otimes \mathbf{M}\right)^{-1 / 2}\right) \mathbf{H}
\end{aligned}
$$

where $\varphi(\alpha, m)$ is given by Eqn. (F.3).

\subsubsection{Analysis of $\mathbf{F}_{2,2}$}

The analysis of $\mathbf{F}_{2,2}$ is similar to the one used for $\mathbf{F}_{1,1}$. Indeed, one has to calculate

$$
\frac{\partial^{2} \ln p\left(\mathbf{c}_{1}, \ldots, \mathbf{c}_{K} ; \boldsymbol{\theta}\right)}{\partial \operatorname{veck}(\mathcal{I} m\{\mathbf{M}\}) \partial \operatorname{veck}^{T}(\mathcal{I} m\{\mathbf{M}\})}=-K \frac{\partial^{2} \ln (|\mathbf{M}|)}{\partial \operatorname{veck}(\mathcal{I} m\{\mathbf{M}\}) \partial \operatorname{veck}^{T}(\mathcal{I} m\{\mathbf{M}\})}
$$




$$
+\sum_{k=1}^{K} \frac{\partial^{2} \ln \left(\left(\mathbf{c}_{k}^{H} \mathbf{M}^{-1} \mathbf{c}_{k}\right)^{\frac{\alpha-m}{2}} K_{m-\alpha}\left(\beta \sqrt{\mathbf{c}_{k}^{H} \mathbf{M}^{-1} \mathbf{c}_{k}}\right)\right)}{\partial \operatorname{veck}(\mathcal{I} m\{\mathbf{M}\}) \partial \operatorname{veck}^{T}(\mathcal{I} m\{\mathbf{M}\})} .
$$

Using Eqn. (A.3), one has

$$
-K \frac{\partial^{2} \ln (|\mathbf{M}|)}{\partial \operatorname{veck}(\mathcal{I} m\{\mathbf{M}\}) \partial \operatorname{veck}^{T}(\mathcal{I} m\{\mathbf{M}\})}=K \mathbf{K}^{T}\left(\mathbf{M}^{T} \otimes \mathbf{M}\right)^{-1} \mathbf{K},
$$

due to Eqn. (28) and due to

$$
\frac{\partial \operatorname{veck}(\mathcal{I} m\{\mathbf{M}\})}{\partial \operatorname{veck}^{T}(\mathcal{I} m\{\mathbf{M}\})}=\mathbf{I} .
$$

By using the same notation as for the derivation of $\mathbf{F}_{1,1}$ and by using Eqn. (36), one obtains for the second term on the right hand side of Eqn. (51)

$$
\begin{aligned}
\frac{\partial^{2} \ln \left(\left(\mathbf{c}_{k}^{H} \mathbf{M}^{-1} \mathbf{c}_{k}\right)^{\frac{\alpha-m}{2}} K_{m-\alpha}\right.}{\partial \operatorname{veck}(\mathcal{I} m\{\mathbf{M}\}) \partial \operatorname{veck}^{T}(\mathcal{I} m\{\mathbf{M}\})} & =2 \beta^{2} d_{1}(z) \mathbf{K}^{T} \mathbf{P}_{1} \mathbf{K}+\beta^{4} d_{2}(z) \mathbf{K}^{T} \mathbf{P}_{2} \mathbf{K}
\end{aligned}
$$

where $\mathbf{P}_{1}, \mathbf{P}_{2}, d_{1}(z)$ and $d_{2}(z)$ are defined by Eqn. (37), Eqn. (38), Eqn. (34), and Eqn. (35), respectively. Therefore, the structure of $\mathbf{F}_{2,2}$ is the same as the structure of $\mathbf{F}_{1,1}$ except that one replaces the matrix $\mathbf{H}$ by the matrix $\mathbf{K}$.

\subsubsection{Analysis of $\mathbf{F}_{1,2}=\mathbf{F}_{2,1}^{T}$}

Due to the structure of Eqn. (A.3) and Eqn. (36), and since the derivation is w.r.t. $\partial \operatorname{vech}(\mathcal{R} e\{\mathbf{M}\})$ and $\partial \operatorname{veck}^{T}(\mathcal{I} m\{\mathbf{M}\})$, it is clear that

$$
\mathbf{F}_{1,2}=\mathbf{F}_{2,1}^{T}=\mathbf{0},
$$

by using Eqn. (28).

This concludes the proof of the CRB derivation.

\section{Simulation results}

In this section, some simulations are provided in order to illustrate the proposed previous results in terms of consistency, bias, and variance analysis 
(throughout the CRB). While no mathematical proof is given in other Sections, we show the efficiency of the MLE meaning that the CRB is achieved by the variance of the MLE.

The results presented are obtained for complex K-distributed clutter with covariance matrix $\mathbf{M}$ randomly chosen.

All the results are presented different values of parameter $\alpha$ and $\beta=2 \sqrt{\alpha}$ following the scenario of [17]. The size of each vector $\mathbf{c}_{k}$ is $m=3$. Remember that the parameter $\alpha$ represents the spikiness of the clutter (when $\alpha$ is high the clutter tends to be Gaussian and, when $\alpha$ is small, the tail of the clutter becomes heavy). The norm used for consitency and bias is the $L^{2}$ norm.

\subsection{Consistency}

Figure 1 presents results of MLE consistency for 1000 Monte Carlo runs per each value of $K$. For that purpose, a plot of $D(\hat{\mathbf{M}}, K)=\|\hat{\mathbf{M}}-\mathbf{M}\|$ versus the number $K$ of $\mathbf{c}_{k}$ 's is presented for each estimate. It can be noticed that the above criterion $D(\hat{\mathbf{M}}, K)$ tends to 0 when $K$ tends to $\infty$ for each estimate. Moreover, note that the parameter $\alpha$ has very few influence on the convergence speed which highlights the robustness of the MLE.

\subsection{Bias}

Figure 2 shows the bias of each estimate for the different values of $\alpha$. The number of Monte Carlo runs is given in the legend of the figure. For that purpose, a plot of the criterion $C(\hat{\mathbf{M}}, K)=\|\overline{\hat{\mathbf{M}}}-\mathbf{M}\|$ versus the number $K$

of $\mathbf{c}_{k}$ 's is presented for each estimate. $\overline{\hat{\mathbf{M}}}$ is defined as the empirical mean of the quantities $\hat{\mathbf{M}}(i)$ obtained from $I$ Monte Carlo runs. For each iteration $i$, a new set of $K$ secondary data $\mathbf{c}_{k}$ is generated to compute $\hat{\mathbf{M}}(i)$. It can be noticed that, as enlightened by the previous theoretical analysis, the bias of $\hat{\mathbf{M}}$ tends to 0 whatever the value of $K$. Furthermore, one sees again the weak influence of the parameter $\alpha$ on the unbiasedness of the MLE.

\subsection{CRB and MSE}

The CRB and empirical variance of the MLE for 10000 Monte Carlo runs are plotted in Figure 3 . For comparison, we also plot the Gaussian CRB (i.e., when $\tau_{k}=1 \forall k$ ) given by Eqn. (25). Although it is not mathematically proved 


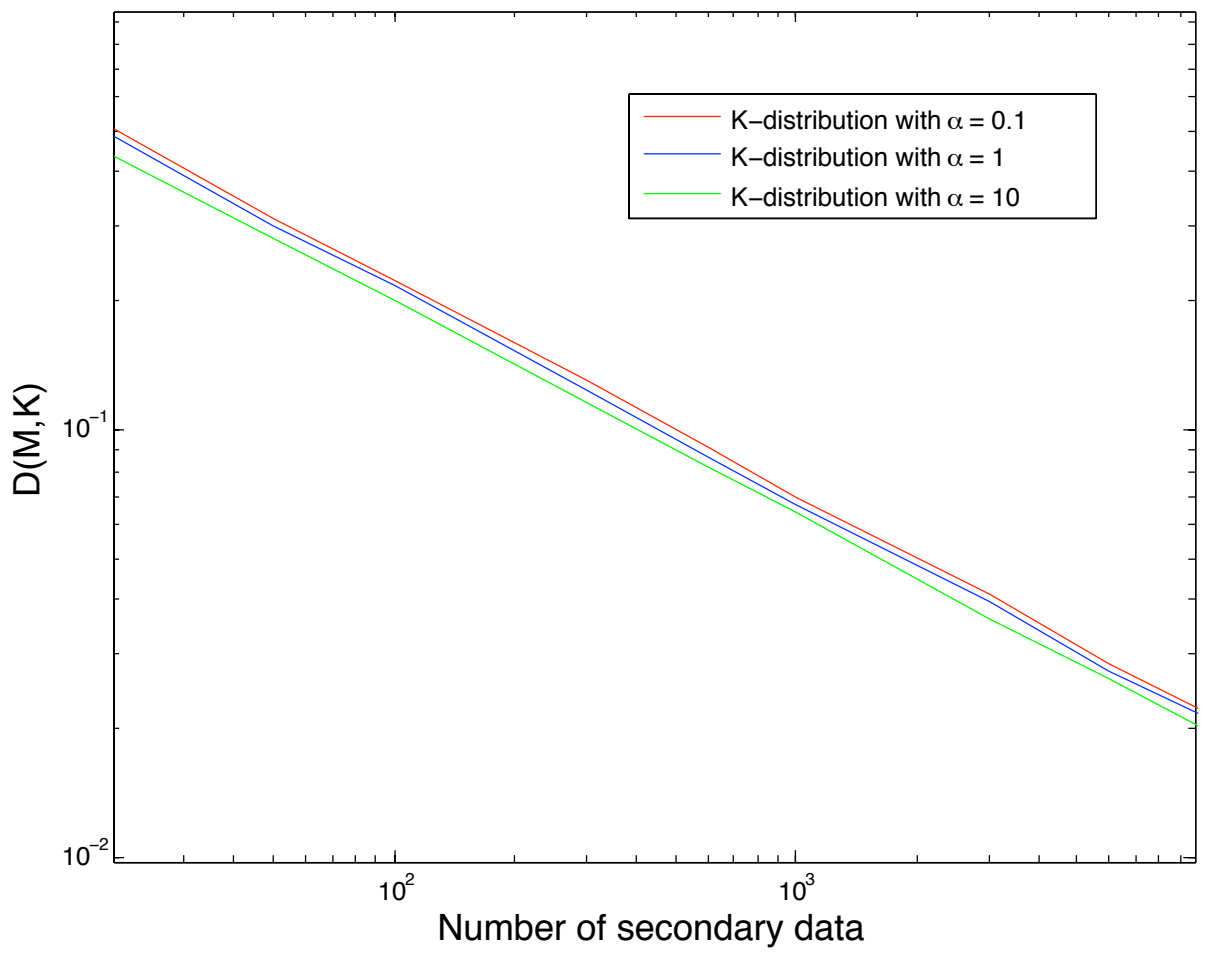

Fig. 1. $D(\hat{\mathbf{M}}, K)$ versus the number of secondary data for different values of $\alpha$.

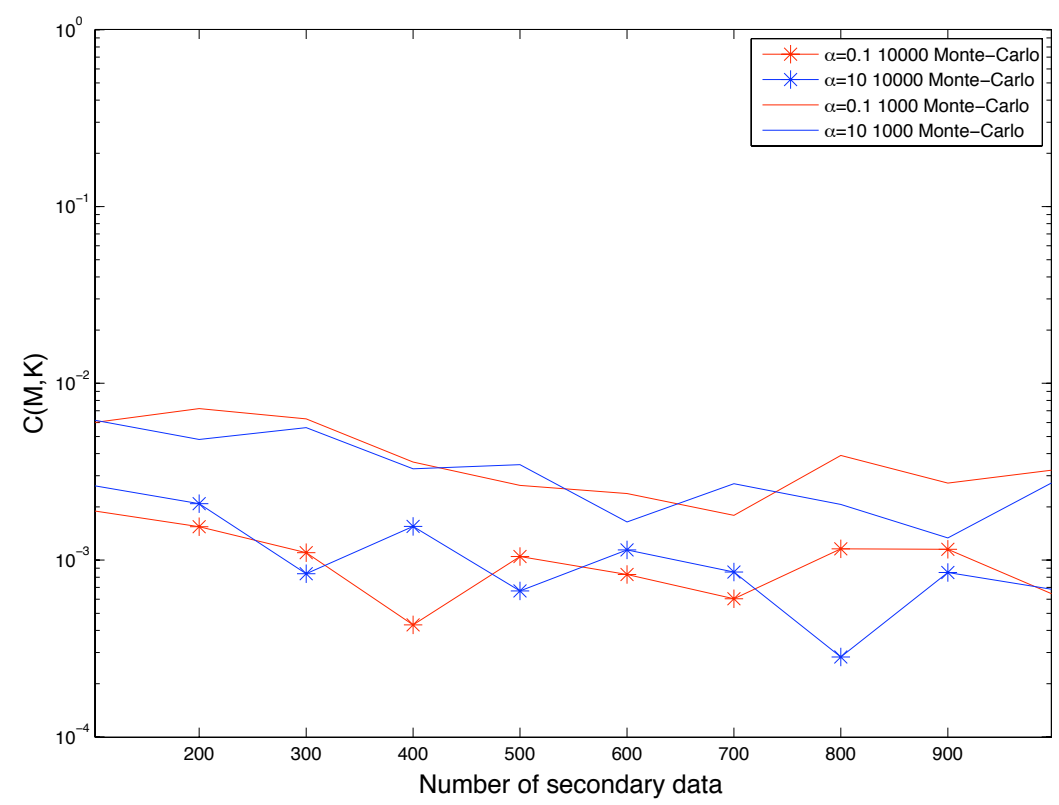

Fig. 2. $C(\hat{\mathbf{M}}, K)$ versus the number of secondary data for different values of $\alpha$.

in this paper, one observes, in Figure 3, the efficiency of the MLE even for impulsive noise ( $\alpha$ small). 


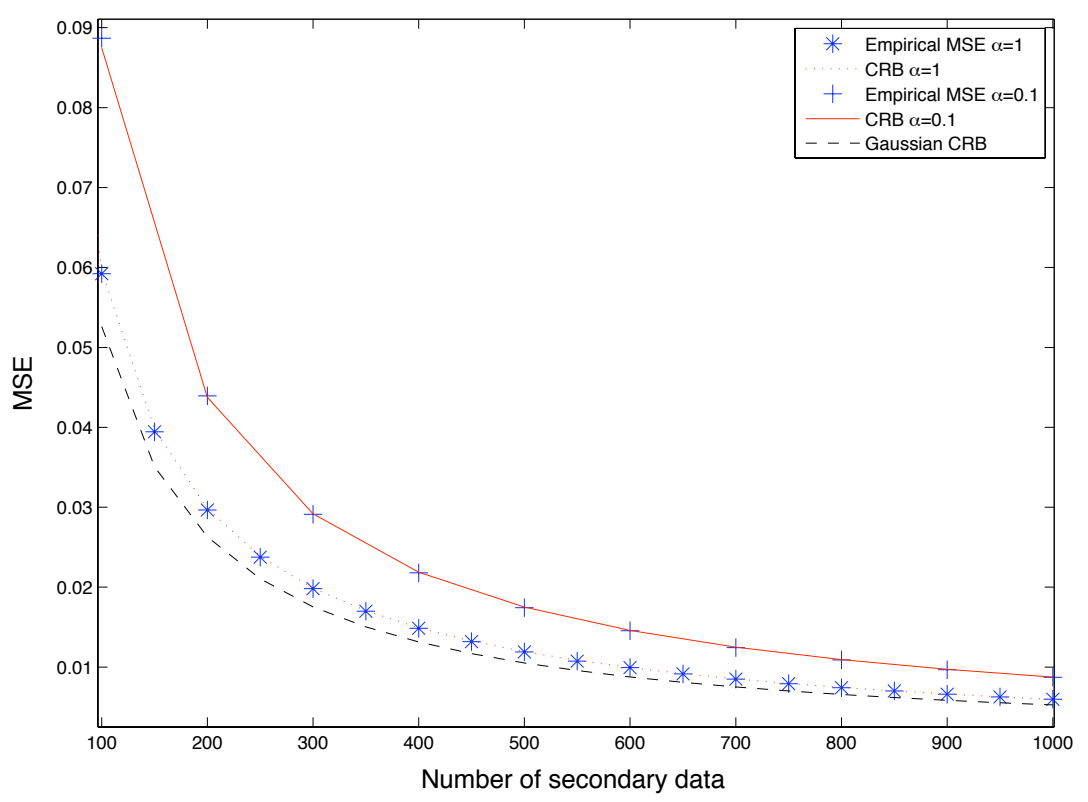

Fig. 3. GCRB, CRB and empirical variance of the MLE for different values of $\alpha$.

\section{Conclusion}

In this paper, a statistical analysis of the maximum likelihood estimator of the covariance matrix of a complex multivariate K-distributed process has been proposed. More particularly, the consistency and the unbiasedness (at finite number of samples) have been proved. In order to analyze the variance of the estimator, the Cramér-Rao lower bound is derived. The Fisher information matrix in this case is simply the Fisher information matrix of the Gaussian case plus a term depending on the tail of the K-distribution. Simulation results have been proposed to illustrate these theoretical analyses. These results have shown the efficiency of the estimator and the weak influence of the spikiness parameter in terms of concistency and bias.

\section{A Derivation of $\partial^{2} \ln \{|\mathbf{M}|\}$}

To find this term and several other, we will use the following results [33]

$$
\begin{aligned}
\partial \operatorname{Tr}(\mathbf{X}) & =\operatorname{Tr}(\partial \mathbf{X}), \\
\partial \operatorname{vec}(\mathbf{X}) & =\operatorname{vec}(\partial \mathbf{X}), \\
\partial \mathbf{A}^{-1} & =-\mathbf{A}^{-1} \partial \mathbf{A} \mathbf{A}^{-1}, \\
\partial|\mathbf{A}| & =|\mathbf{A}| \operatorname{Tr}\left(\mathbf{A}^{-1} \partial \mathbf{A}\right),
\end{aligned}
$$




$$
\begin{array}{rlrl}
\partial(\mathbf{A} \& \mathbf{B}) & =\partial(\mathbf{A}) \& \mathbf{B}+\mathbf{A} \& \partial(\mathbf{B}), \text { where } \&=\times \text { or } \otimes(\mathrm{A} .1 \mathrm{e}) \\
\partial \ln (|\mathbf{M}|) & =\operatorname{Tr}\left(\mathbf{M}^{-1} \partial \mathbf{M}\right), & (\mathrm{A} .1 \mathrm{f}) \\
\partial(\partial \mathbf{M}) & =\mathbf{0}, & (\mathrm{A} .1 \mathrm{~g}) \\
\operatorname{Tr}(\mathbf{A B}) & =\operatorname{Tr}(\mathbf{B A}) & (\mathrm{A} .1 \mathrm{~h}) \\
\mathbf{M}^{-1} \otimes \mathbf{M}^{-1} & =(\mathbf{M} \otimes \mathbf{M})^{-1}, \\
\operatorname{Tr}\left(\mathbf{A}^{H} \mathbf{B}\right) & =\operatorname{vec}^{H}(\mathbf{A}) \operatorname{vec}(\mathbf{B}), & (\mathrm{A} .1 \mathrm{j}) \\
\operatorname{vec}(\mathbf{A B C}) & =\left(\mathbf{C}^{T} \otimes \mathbf{A}\right) \operatorname{vec}(\mathbf{B}), &
\end{array}
$$

By using these properties, one has

$$
\begin{aligned}
\partial \ln (|\mathbf{M}|) & =\operatorname{Tr}\left(\mathbf{M}^{-1}(\partial \mathbf{M})\right) \quad \text { from }(\text { A.1f }) \\
\partial^{2} \ln (|\mathbf{M}|) & =-\operatorname{Tr}\left(\mathbf{M}^{-1}(\partial \mathbf{M}) \mathbf{M}^{-1} \partial \mathbf{M}\right) \quad \text { from }(\mathrm{A} .1 \mathrm{a})(\mathrm{A} .1 \mathrm{e})(\mathrm{A} .1 \mathrm{~g})(\mathrm{A} .1 \mathrm{c}) \\
& =-\operatorname{vec}^{H}\left(\mathbf{M}^{-1}(\partial \mathbf{M}) \mathbf{M}^{-1}\right) \operatorname{vec}(\partial \mathbf{M}) \quad \text { from }(\mathrm{A} .1 \mathrm{j}) \\
& =-\operatorname{vec}^{H}(\partial \mathbf{M})\left(\mathbf{M}^{-T} \otimes \mathbf{M}^{-1}\right)^{H} \operatorname{vec}(\partial \mathbf{M}) \quad \text { from }(\mathrm{A} .1 \mathrm{k}) \\
& =-\partial \operatorname{vec}^{H}(\mathbf{M})\left(\mathbf{M}^{T} \otimes \mathbf{M}\right)^{-1} \partial \operatorname{vec}(\mathbf{M}) \quad \text { from }(\mathrm{A} .1 \mathrm{~b})(\mathrm{A} .1 \mathrm{i})
\end{aligned}
$$

By letting $\mathbf{M}=\mathcal{R} e\{\mathbf{M}\}+i \mathcal{I} m\{\mathbf{M}\}$ in Eqn.(A.3), one has

$$
\begin{aligned}
\partial^{2} \ln (|\mathbf{M}|)= & -\partial \operatorname{vec}^{H}(\mathcal{R} e\{\mathbf{M}\}+i \mathcal{I} m\{\mathbf{M}\})\left(\mathbf{M}^{T} \otimes \mathbf{M}\right)^{-1} \partial \operatorname{vec}(\mathcal{R} e\{\mathbf{M}\}+i \mathcal{I} m\{\mathbf{M}\}) \\
= & -\partial \operatorname{vec}^{H}(\mathcal{R} e\{\mathbf{M}\})\left(\mathbf{M}^{T} \otimes \mathbf{M}\right)^{-1} \partial \operatorname{vec}(\mathcal{R} e\{\mathbf{M}\}) \\
& -\partial \operatorname{vec}^{H}(i \mathcal{I} m\{\mathbf{M}\})\left(\mathbf{M}^{T} \otimes \mathbf{M}\right)^{-1} \partial \operatorname{vec}(\mathcal{R} e\{\mathbf{M}\}) \\
& -\partial \operatorname{vec}^{H}(\mathcal{R} e\{\mathbf{M}\})\left(\mathbf{M}^{T} \otimes \mathbf{M}\right)^{-1} \partial \operatorname{vec}(i \mathcal{I} m\{\mathbf{M}\}) \\
& -\partial \operatorname{vec}^{H}(i \mathcal{I} m\{\mathbf{M}\})\left(\mathbf{M}^{T} \otimes \mathbf{M}\right)^{-1} \partial \operatorname{vec}(i \mathcal{I} m\{\mathbf{M}\}) .
\end{aligned}
$$

Since vec ${ }^{H}(i \mathcal{I} m\{\mathbf{M}\})=-i \operatorname{vec}^{T}(\mathcal{I} m\{\mathbf{M}\})$ and $\operatorname{vec}^{H}(\mathcal{R} e\{\mathbf{M}\})=\operatorname{vec}^{T}(\mathcal{R} e\{\mathbf{M}\})$, $\partial^{2} \ln (|\mathbf{M}|)$ is reduced to

$$
\begin{aligned}
\partial^{2} \ln (|\mathbf{M}|)= & -\partial \operatorname{vec}^{T}(\mathcal{R} e\{\mathbf{M}\})\left(\mathbf{M}^{T} \otimes \mathbf{M}\right)^{-1} \partial \operatorname{vec}(\mathcal{R} e\{\mathbf{M}\}) \\
& -\partial \operatorname{vec}^{T}(\mathcal{I} m\{\mathbf{M}\})\left(\mathbf{M}^{T} \otimes \mathbf{M}\right)^{-1} \partial \operatorname{vec}(\mathcal{I} m\{\mathbf{M}\}) \\
= & -\partial \operatorname{vech}^{T}(\mathcal{R} e\{\mathbf{M}\}) \mathbf{H}^{T}\left(\mathbf{M}^{T} \otimes \mathbf{M}\right)^{-1} \mathbf{H} \partial \operatorname{vech}(\mathcal{R} e\{\mathbf{M}\}) \\
& -\partial \operatorname{veck}^{T}(\mathcal{I} m\{\mathbf{M}\}) \mathbf{K}^{T}\left(\mathbf{M}^{T} \otimes \mathbf{M}\right)^{-1} \mathbf{K} \partial \operatorname{veck}(\mathcal{I} m\{\mathbf{M}\} A, 3)
\end{aligned}
$$


where the matrix $\mathbf{H}$ and $\mathbf{K}$ are constant transformation matrices filled with ones and zeros such that $\operatorname{vec}(\mathbf{A})=\mathbf{H} \operatorname{vech}(\mathbf{A})$ and $\operatorname{vec}(\mathbf{A})=\mathbf{K} \operatorname{veck}(\mathbf{A})$.

\section{B Derivation of $\partial z$ and $\partial^{2} z$}

By using the properties from Eqn.(A.1a) to Eqn.(A.1k), one has for $\partial z$

$$
\begin{aligned}
\partial z & =\beta^{2} \partial\left(\mathbf{c}_{k}^{H} \mathbf{M}^{-1} \mathbf{c}_{k}\right)=\beta^{2} \partial \operatorname{Tr}\left(\mathbf{c}_{k}^{H} \mathbf{M}^{-1} \mathbf{c}_{k}\right) \\
& =\beta^{2} \operatorname{Tr}\left(\mathbf{c}_{k}^{H} \partial\left(\mathbf{M}^{-1}\right) \mathbf{c}_{k}\right) \quad \text { from (A.1a) } \\
& =-\beta^{2} \operatorname{Tr}\left(\mathbf{c}_{k}^{H} \mathbf{M}^{-1} \partial \mathbf{M} \mathbf{M}^{-1} \mathbf{c}_{k}\right) \quad \text { from (A.1c) } \\
& =-\beta^{2} \operatorname{Tr}\left(\partial \mathbf{M} \mathbf{M}^{-1} \mathbf{c}_{k} \mathbf{c}_{k}^{H} \mathbf{M}^{-1}\right) \quad \text { from }(\mathrm{A} .1 \mathrm{~h}) \\
& =-\beta^{2} \operatorname{vec}^{H}(\partial \mathbf{M}) \operatorname{vec}\left(\mathbf{M}^{-1} \mathbf{c}_{k} \mathbf{c}_{k}^{H} \mathbf{M}^{-1}\right) \quad \text { from (A.1j) } \\
& =-\beta^{2} \partial \operatorname{vec}^{H}(\mathbf{M})\left(\mathbf{M}^{T} \otimes \mathbf{M}\right)^{-1} \operatorname{vec}\left(\mathbf{c}_{k} \mathbf{c}_{k}^{H}\right) \quad \text { from (A.1b)(A.1k)(A.1i). }
\end{aligned}
$$

By letting $\mathbf{M}=\mathcal{R} e\{\mathbf{M}\}+i \mathcal{I} m\{\mathbf{M}\}$, one obtains

$$
\begin{aligned}
\partial z= & -\beta^{2} \partial \operatorname{vec}^{T}(\mathcal{R} e\{\mathbf{M}\})\left(\mathbf{M}^{T} \otimes \mathbf{M}\right)^{-1} \operatorname{vec}\left(\mathbf{c}_{k} \mathbf{c}_{k}^{H}\right) \\
& +i \beta^{2} \partial \operatorname{vec}^{T}(\mathcal{I} m\{\mathbf{M}\})\left(\mathbf{M}^{T} \otimes \mathbf{M}\right)^{-1} \operatorname{vec}\left(\mathbf{c}_{k} \mathbf{c}_{k}^{H}\right) \\
= & -\beta^{2} \partial \operatorname{vech}^{T}(\mathcal{R} e\{\mathbf{M}\}) \mathbf{H}^{T}\left(\mathbf{M}^{T} \otimes \mathbf{M}\right)^{-1} \operatorname{vec}\left(\mathbf{c}_{k} \mathbf{c}_{k}^{H}\right) \\
& +i \beta^{2} \partial \operatorname{veck}^{T}(\mathcal{I} m\{\mathbf{M}\}) \mathbf{K}^{T}\left(\mathbf{M}^{T} \otimes \mathbf{M}\right)^{-1} \operatorname{vec}\left(\mathbf{c}_{k} \mathbf{c}_{k}^{H}\right) .
\end{aligned}
$$

Concerning $\partial^{2} z$, one has

$$
\begin{aligned}
\partial z & =-\beta^{2} \operatorname{Tr}\left(\mathbf{c}_{k}^{H} \mathbf{M}^{-1} \partial \mathbf{M} \mathbf{M}^{-1} \mathbf{c}_{k}\right) \\
\partial^{2} z & =-\beta^{2} \operatorname{Tr}\left(\mathbf{c}_{k}^{H} \partial\left(\mathbf{M}^{-1} \partial \mathbf{M} \mathbf{M}^{-1}\right) \mathbf{c}_{k}\right) \quad \text { from (A.1a) } \\
& =-\beta^{2} \operatorname{Tr}\left(\mathbf{c}_{k}^{H} \partial\left(\mathbf{M}^{-1}\right) \partial \mathbf{M} \mathbf{M}^{-1} \mathbf{c}_{k}+\mathbf{c}_{k}^{H} \mathbf{M}^{-1} \partial \mathbf{M} \partial\left(\mathbf{M}^{-1}\right) \mathbf{c}_{k}\right) \quad \text { from (A.1e)(A.1g) } \\
& =2 \beta^{2} \operatorname{Tr}\left(\partial \mathbf{M} \mathbf{M}^{-1} \partial \mathbf{M} \mathbf{M}^{-1} \mathbf{c}_{k} \mathbf{c}_{k}^{H} \mathbf{M}^{-1}\right) \quad \text { from (A.1c)(A.1h) } \\
& =2 \beta^{2} \partial \operatorname{vec}^{H}(\mathbf{M})\left(\left(\mathbf{M}^{-T} \mathbf{c}_{k}^{*} \mathbf{c}_{k}^{T} \mathbf{M}^{-T}\right) \otimes \mathbf{M}^{-1}\right) \partial \operatorname{vec}(\mathbf{M}) \quad \text { from (A.1j)(A.1k)(A.1b) }
\end{aligned}
$$


By letting $\mathbf{M}=\mathcal{R} e\{\mathbf{M}\}+i \mathcal{I} m\{\mathbf{M}\}$, one obtains

$$
\begin{gathered}
\partial^{2} z=2 \beta^{2} \partial \operatorname{vech}^{T}(\mathcal{R} e\{\mathbf{M}\}) \mathbf{H}^{T}\left(\left(\mathbf{M}^{-T} \mathbf{c}_{k}^{*} \mathbf{c}_{k}^{T} \mathbf{M}^{-T}\right) \otimes \mathbf{M}^{-1}\right) \mathbf{H} \partial \operatorname{vech}(\mathcal{R} e\{\mathbf{M}\}) \\
+2 \beta^{2} \partial \operatorname{veck}^{T}(\mathcal{I} m\{\mathbf{M}\}) \mathbf{K}^{T}\left(\left(\mathbf{M}^{-T} \mathbf{c}_{k}^{*} \mathbf{c}_{k}^{T} \mathbf{M}^{-T}\right) \otimes \mathbf{M}^{-1}\right) \mathbf{K} \partial \operatorname{veck}(\mathcal{I} m\{\mathbf{M}\}) .
\end{gathered}
$$

\section{Derivation of $\frac{\partial f(z)}{\partial z}$ and $\frac{\partial^{2} f(z)}{\partial z^{2}}$}

Concerning the first derivative of $f(z)$, one has

$$
\begin{aligned}
\frac{\partial f(z)}{\partial z} & =\frac{\partial}{\partial z} \ln \left(\left(z / \beta^{2}\right)^{\frac{\alpha-m}{2}} K_{m-\alpha}(\sqrt{z})\right) \\
& =\frac{\alpha-m}{2 z}+\frac{1}{K_{m-\alpha}(\sqrt{z})} \frac{\partial K_{m-\alpha}(\sqrt{z})}{\partial z} .
\end{aligned}
$$

Since $\frac{\partial K_{\nu}(y)}{\partial y}=-K_{\nu+1}(y)+\frac{\nu}{y} K_{\nu}(y)$ [34]. It follows that

$$
\frac{\partial K_{m-\alpha}(\sqrt{z})}{\partial z}=-\frac{1}{2 \sqrt{z}} K_{m-\alpha+1}(\sqrt{z})+\frac{m-\alpha}{2 z} K_{m-\alpha}(\sqrt{z}) .
$$

Plugging Eqn. (C.2) in Eqn. (C.1), one obtains

$$
\frac{\partial f(z)}{\partial z}=-\frac{1}{2 \sqrt{z}} \frac{K_{m-\alpha+1}(\sqrt{z})}{K_{m-\alpha}(\sqrt{z})} .
$$

Concerning the second derivative of $f(z)$, one has

$$
\begin{aligned}
\frac{\partial^{2} f(z)}{\partial z^{2}} & =-\frac{1}{2} \frac{\partial}{\partial z}\left(\frac{1}{\sqrt{z}} \frac{K_{m-\alpha+1}(\sqrt{z})}{K_{m-\alpha}(\sqrt{z})}\right) \\
& =\frac{1}{4 z^{3 / 2}} \frac{K_{m-\alpha+1}(\sqrt{z})}{K_{m-\alpha}(\sqrt{z})}-\frac{1}{4 z}\left(\left.\frac{\partial}{\partial y} \frac{K_{m-\alpha+1}(y)}{K_{m-\alpha}(y)}\right|_{y=\sqrt{z}}\right)
\end{aligned}
$$

Since $\frac{\partial K_{\nu}(y)}{\partial y}=-K_{\nu-1}(y)-\frac{\nu}{y} K_{\nu}(y)$ [34]. It follows that

$$
\left.\frac{\partial}{\partial y} \frac{K_{m-\alpha+1}(y)}{K_{m-\alpha}(y)}\right|_{y=\sqrt{z}}=-\frac{\left(K_{m-\alpha}(\sqrt{z})+\frac{m-\alpha+1}{\sqrt{z}} K_{m-\alpha+1}(\sqrt{z})\right) K_{m-\alpha}(\sqrt{z})}{K_{m-\alpha}^{2}(\sqrt{z})}
$$




$$
+\frac{K_{m-\alpha+1}(\sqrt{z})\left(K_{m-\alpha-1}(\sqrt{z})+\frac{m-\alpha}{\sqrt{z}} K_{m-\alpha}(\sqrt{z})\right)}{K_{m-\alpha}^{2}(\sqrt{z})} .
$$

Plugging Eqn. (C.5) in Eqn. (C.4), one obtains

$$
\frac{\partial^{2} f(z)}{\partial z^{2}}=\frac{1}{4 z}+\frac{1}{2 z^{3 / 2}} \frac{K_{m-\alpha+1}(\sqrt{z})}{K_{m-\alpha}(\sqrt{z})}-\frac{1}{4 z} \frac{K_{m-\alpha+1}(\sqrt{z}) K_{m-\alpha-1}(\sqrt{z})}{K_{m-\alpha}^{2}(\sqrt{z})} .
$$

\section{Derivation of matrix $\Gamma$}

The matrix $\boldsymbol{\Gamma}$ is given by

$$
\begin{aligned}
\boldsymbol{\Gamma} & =E\left[\frac{1}{\sqrt{z}} \frac{K_{m-\alpha+1}(\sqrt{z})}{K_{m-\alpha}(\sqrt{z})} \mathbf{c}_{k}^{*} \mathbf{c}_{k}^{T}\right] \\
& =\frac{\Gamma(\alpha-1)}{2 \Gamma(\alpha)} E^{T}\left[\mathbf{c}_{k} \mathbf{c}_{k}^{H}\right],
\end{aligned}
$$

where the last expectation is taken under the distribution

$$
p\left(\mathbf{c}_{k}\right)=\frac{\beta^{\alpha+m-1}|\mathbf{M}|^{-1}}{2^{\alpha+m-2} \pi^{m} \Gamma(\alpha-1)}\left(\mathbf{c}_{k}^{H} \mathbf{M}^{-1} \mathbf{c}_{k}\right)^{\frac{\alpha-m-1}{2}} K_{m-\alpha+1}\left(\beta \sqrt{\mathbf{c}_{k}^{H} \mathbf{M}^{-1} \mathbf{c}_{k}}\right),
$$

which is a complex K-distribution $\mathcal{K}_{m}\left(\alpha-1,(2 / \beta)^{2}, \mathbf{M}\right)$. Then

$\boldsymbol{\Gamma}=\frac{1}{2(\alpha-1)} E^{T}\left[\mathbf{c}_{k} \mathbf{c}_{k}^{H}\right]=\frac{1}{2(\alpha-1)} E^{T}\left[\tau_{k} \mathbf{x}_{k} \mathbf{x}_{k}^{H}\right]=\frac{1}{2(\alpha-1)} E\left[\tau_{k}\right] E^{T}\left[\mathbf{x}_{k} \mathbf{x}_{k}^{H}\right]$,

where $E\left[\tau_{k}\right]=(\alpha-1)\left(\frac{2}{\beta}\right)^{2}$ since $\tau_{k}$ follows a Gamma distribution $\mathcal{G}\left(\alpha-1,(2 / \beta)^{2}\right)$ and $E^{T}\left[\mathbf{x}_{k} \mathbf{x}_{k}^{H}\right]=\mathbf{M}^{T}$ since $\mathbf{x}_{k}$ is a complex normal random vector (independent of $\tau_{k}$ ) with zero mean and covariance matrix M. Consequently, $\boldsymbol{\Gamma}=\frac{2}{\beta^{2}} \mathbf{M}^{T}$.

E Derivation of $E\left[\frac{\operatorname{vec}\left(\mathbf{c}_{k} \mathbf{c}_{k}^{H}\right) \mathbf{v e c}^{H}\left(\mathbf{c}_{k} \mathbf{c}_{k}^{H}\right)}{\mathbf{c}_{k}^{H} \mathbf{M}^{-1} \mathbf{c}_{k}}\right]$

In this Appendix, we derive the expression of $E\left[\frac{\operatorname{vec}\left(\mathbf{c}_{k} \mathbf{c}_{k}^{H}\right) \operatorname{vec}^{H}\left(\mathbf{c}_{k} \mathbf{c}_{k}^{H}\right)}{\mathbf{c}_{k}^{H} \mathbf{M}^{-1} \mathbf{c}_{k}}\right]$ where $\mathbf{c}_{k} \sim \mathcal{K}_{m}\left(\alpha,(2 / \beta)^{2}, \mathbf{M}\right)$. The case where $\mathbf{c}_{k} \sim \mathcal{K}_{m}\left(\alpha-1,(2 / \beta)^{2}, \mathbf{M}\right)$ will 
be, of course, straightforward. Let us set the following change of variable: $\mathbf{c}_{k}=\mathbf{M}^{1 / 2} \mathbf{y}_{k}$. One obtains from Eqn. (A.1k)

$$
\begin{aligned}
& E\left[\frac{\operatorname{vec}\left(\mathbf{c}_{k} \mathbf{c}_{k}^{H}\right) \operatorname{vec}^{H}\left(\mathbf{c}_{k} \mathbf{c}_{k}^{H}\right)}{\mathbf{c}_{k}^{H} \mathbf{M}^{-1} \mathbf{c}_{k}}\right] \\
& =\left(\mathbf{M}^{T / 2} \otimes \mathbf{M}^{1 / 2}\right) E\left[\frac{\operatorname{vec}\left(\mathbf{y}_{k} \mathbf{y}_{k}^{H}\right) \operatorname{vec}^{H}\left(\mathbf{y}_{k} \mathbf{y}_{k}^{H}\right)}{\mathbf{y}_{k}^{H} \mathbf{y}_{k}}\right]\left(\mathbf{M}^{T / 2} \otimes \mathbf{M}^{1 / 2}\right)
\end{aligned}
$$

where $\mathbf{y}_{k} \sim \mathcal{K}_{m}\left(\alpha,(2 / \beta)^{2}, \mathbf{I}\right)$. Since $\mathbf{y}_{k}=\sqrt{\tau_{k}} \mathbf{x}_{k}$, where $\tau_{k} \sim \mathcal{G}\left(\alpha,(2 / \beta)^{2}\right)$ is independent of $\mathbf{x}_{k} \sim \mathcal{C N}(\mathbf{0}, \mathbf{I})$, one has

$$
E\left[\frac{\operatorname{vec}\left(\mathbf{c}_{k} \mathbf{c}_{k}^{H}\right) \operatorname{vec}^{H}\left(\mathbf{c}_{k} \mathbf{c}_{k}^{H}\right)}{\mathbf{c}_{k}^{H} \mathbf{M}^{-1} \mathbf{c}_{k}}\right]=\left(\mathbf{M}^{T / 2} \otimes \mathbf{M}^{1 / 2}\right) E\left[\tau_{k}\right] \mathbf{R}\left(\mathbf{M}^{T / 2} \otimes \mathbf{M}^{1 / 2}\right)
$$

where $E\left[\tau_{k}\right]=\alpha\left(\frac{2}{\beta}\right)^{2}$ and where

$$
\mathbf{R}=E\left[\frac{\operatorname{vec}\left(\mathbf{x}_{k} \mathbf{x}_{k}^{H}\right) \operatorname{vec}^{H}\left(\mathbf{x}_{k} \mathbf{x}_{k}^{H}\right)}{\mathbf{x}_{k}^{H} \mathbf{x}_{k}}\right]
$$

Let us set $x_{k}^{(j)}=\sqrt{\rho_{j}^{2}} \exp \left(i \theta_{j}\right)$ for $j=1, \ldots, m$. Note that $\rho_{j}^{2} \sim \chi^{2}(2)$ is independent of $\theta_{j} \sim \mathcal{U}_{[0,2 \pi]}$. Consequently, the elements of the matrix $\mathbf{R}$ can be rewritten as

$$
R_{k, l}=E\left[\frac{\sqrt{\rho_{p}^{2} \rho_{q}^{2} \rho_{p^{\prime}}^{2} \rho_{q^{\prime}}^{2}}}{\sum_{j=1}^{m} \rho_{j}^{2}}\right] E\left[\exp \left(i\left(\theta_{p}-\theta_{p^{\prime}}+\theta_{q^{\prime}}-\theta_{q}\right)\right)\right]
$$

since

$$
\mathbf{x}_{k}^{H} \mathbf{x}_{k}=\sum_{j=1}^{m} \rho_{j}^{2} \text { and }\left[\operatorname{vec}\left(\mathbf{x}_{k} \mathbf{x}_{k}^{H}\right)\right]_{n}=\sqrt{\rho_{p}^{2} \rho_{p^{\prime}}^{2}} \exp \left(i\left(\theta_{p}-\theta_{p^{\prime}}\right)\right)
$$

Note that $E\left[\exp \left(i\left(\theta_{p}-\theta_{p^{\prime}}+\theta_{q^{\prime}}-\theta_{q}\right)\right)\right] \neq 0$ if and only if

(1) $p=p^{\prime}=q=q^{\prime}$, i.e., $l=k=p+m(p-1)$,

(2) $p=p^{\prime}, q=q^{\prime}$ and $p \neq q$, i.e., $l=k=p+m(q-1)$,

(3) $p=q, p^{\prime}=q^{\prime}$ and $p \neq p^{\prime}$, i.e., $l=p^{\prime}+m\left(p^{\prime}-1\right)$ and $k=p+m(p-1)$.

Consequently, the non-zero elements of $R_{k, l}$ are given by 
(1) $R_{p+m(p-1), p+m(p-1)}=E\left[\frac{\left(\rho_{p}^{2}\right)^{2}}{\sum_{j=1}^{m} \rho_{j}^{2}}\right]=\frac{4}{m+1}$,

(2) $R_{p+m(q-1), p+m(q-1)}=E\left[\begin{array}{c}\rho_{p}^{2} \rho_{p^{\prime}}^{2} \\ \sum_{j=1}^{m} \rho_{j}^{2}\end{array}\right]=\frac{2}{m+1}$,

(3) $R_{p+m(p-1), p^{\prime}+m\left(p^{\prime}-1\right)}=E\left[\frac{\rho_{p}^{2} \rho_{q}^{2}}{\sum_{j=1}^{m} \rho_{j}^{2}}\right]=\frac{2}{m+1}$,

and the matrix $E\left[\frac{\operatorname{vec}\left(\mathbf{c}_{k} \mathbf{c}_{k}^{H}\right) \operatorname{vec}^{H}\left(\mathbf{c}_{k} \mathbf{c}_{k}^{H}\right)}{\mathbf{c}_{k}^{H} \mathbf{M}^{-1} \mathbf{c}_{k}}\right]$ can be written

$$
\begin{aligned}
& E\left[\frac{\operatorname{vec}\left(\mathbf{c}_{k} \mathbf{c}_{k}^{H}\right) \operatorname{vec}^{H}\left(\mathbf{c}_{k} \mathbf{c}_{k}^{H}\right)}{\mathbf{c}_{k}^{H} \mathbf{M}^{-1} \mathbf{c}_{k}}\right] \\
= & \frac{2 \alpha}{m+1}\left(\frac{2}{\beta}\right)^{2}\left(\mathbf{M}^{T / 2} \otimes \mathbf{M}^{1 / 2}\right)\left(\mathbf{I}+\operatorname{vec}(\mathbf{I}) \operatorname{vec}^{T}(\mathbf{I})\right)\left(\mathbf{M}^{T / 2} \otimes \mathbf{M}^{1 / 2}\right) .
\end{aligned}
$$

In the same way, the expression of $E\left[\frac{\operatorname{vec}\left(\mathbf{c}_{k} \mathbf{c}_{k}^{H}\right) \operatorname{vec}{ }^{H}\left(\mathbf{c}_{k} \mathbf{c}_{k}^{H}\right)}{\mathbf{c}_{k}^{H} \mathbf{M}^{-1} \mathbf{c}_{k}}\right]$ where $\mathbf{c}_{k} \sim \mathcal{K}_{m}\left(\alpha-1,(2 / \beta)^{2}, \mathbf{M}\right)$ is given by

$$
\begin{aligned}
& E\left[\frac{\operatorname{vec}\left(\mathbf{c}_{k} \mathbf{c}_{k}^{H}\right) \operatorname{vec}^{H}\left(\mathbf{c}_{k} \mathbf{c}_{k}^{H}\right)}{\mathbf{c}_{k}^{H} \mathbf{M}^{-1} \mathbf{c}_{k}}\right] \\
& =\frac{2(\alpha-1)}{m+1}\left(\frac{2}{\beta}\right)^{2}\left(\mathbf{M}^{T / 2} \otimes \mathbf{M}^{1 / 2}\right)\left(\mathbf{I}+\operatorname{vec}(\mathbf{I}) \operatorname{vec}^{T}(\mathbf{I})\right)\left(\mathbf{M}^{T / 2} \otimes \mathbf{M}^{1 / 2}\right)
\end{aligned}
$$

\section{F Analysis of $\Upsilon$}

Let us set the following change of variable: $\mathbf{c}_{k}=\mathbf{M}^{1 / 2} \sqrt{\tau_{k}} \mathbf{x}_{k}$, where $\tau_{k} \sim$ $\mathcal{G}\left(\alpha,(2 / \beta)^{2}\right)$ is independent of $\mathbf{x}_{k} \sim \mathcal{C N}(\mathbf{0}, \mathbf{I})$, one has

$$
\Upsilon=\frac{1}{\beta^{2}}\left(\mathbf{M}^{T / 2} \otimes \mathbf{M}^{1 / 2}\right) \tilde{\Upsilon}\left(\mathbf{M}^{T / 2} \otimes \mathbf{M}^{1 / 2}\right)
$$


where

$\tilde{\boldsymbol{\Upsilon}}=E\left[\tau_{k} \frac{K_{m-\alpha-1}\left(\beta \sqrt{\tau_{k} \mathbf{x}_{k}^{H} \mathbf{x}_{k}}\right) K_{m-\alpha+1}\left(\beta \sqrt{\tau_{k} \mathbf{x}_{k}^{H} \mathbf{x}_{k}}\right)}{K_{m-\alpha}^{2}\left(\beta \sqrt{\tau_{k} \mathbf{x}_{k}^{H} \mathbf{x}_{k}}\right)} \frac{\operatorname{vec}\left(\mathbf{x}_{k} \mathbf{x}_{k}^{H}\right) \operatorname{vec}^{H}\left(\mathbf{x}_{k} \mathbf{x}_{k}^{H}\right)}{\mathbf{x}_{k}^{H} \mathbf{x}_{k}}\right]$.

Let us set $x_{k}^{(j)}=\sqrt{\rho_{j}^{2}} \exp \left(i \theta_{j}\right)$ for $j=1, \ldots, m . \rho_{j}^{2} \sim \chi^{2}(2)$ is independent of $\theta_{j} \sim \mathcal{U}_{[0,2 \pi]}$. Consequently, due to Eqn. (E.5), the elements of the matrix $\tilde{\Upsilon}$ can be rewritten as

$$
\begin{aligned}
\tilde{\Upsilon}_{k, l}= & E\left[\tau_{k^{\prime}} \frac{K_{m-\alpha-1}\left(\beta \sqrt{\tau_{k^{\prime}} \sum_{j=1}^{m} \rho_{j}^{2}}\right) K_{m-\alpha+1}\left(\beta \sqrt{\tau_{k^{\prime}} \sum_{j=1}^{m} \rho_{j}^{2}}\right)}{K_{m-\alpha}^{2}\left(\beta \sqrt{\tau_{k^{\prime}} \sum_{j=1}^{m} \rho_{j}^{2}}\right)} \frac{\sqrt{\rho_{p}^{2} \rho_{q}^{2} \rho_{p^{\prime}}^{2} \rho_{q^{\prime}}^{2}}}{\sum_{j=1}^{m} \rho_{j}^{2}}\right] \\
& \times E\left[\exp \left(i\left(\theta_{p}-\theta_{p^{\prime}}+\theta_{q^{\prime}}-\theta_{q}\right)\right)\right] .
\end{aligned}
$$

As before, $E\left[\exp \left(i\left(\theta_{p}-\theta_{p^{\prime}}+\theta_{q^{\prime}}-\theta_{q}\right)\right)\right] \neq 0$ if and only if

(1) $p=p^{\prime}=q=q^{\prime}$, i.e., $l=k=p+m(p-1)$,

(2) $p=p^{\prime}, q=q^{\prime}$ and $p \neq q$, i.e., $l=k=p+m(q-1)$,

(3) $p=q, p^{\prime}=q^{\prime}$ and $p \neq p^{\prime}$, i.e., $l=p^{\prime}+m\left(p^{\prime}-1\right)$ and $k=p+m(p-1)$.

Consequently, the non-zero elements of $\tilde{\Upsilon}_{k, l}$ are given by

(1) $\tilde{\Upsilon}_{p+m(p-1), p+m(p-1)}=E\left[\tau_{k^{\prime}} \frac{K_{m-\alpha-1}\left(\beta \sqrt{\tau_{k^{\prime}} \sum_{j=1}^{m} \rho_{j}^{2}}\right) K_{m-\alpha+1}\left(\beta \sqrt{\tau_{k^{\prime}} \sum_{j=1}^{m} \rho_{j}^{2}}\right)}{K_{m-\alpha}^{2}\left(\beta \sqrt{\tau_{k^{\prime}} \sum_{j=1}^{m} \rho_{j}^{2}}\right)} \frac{\left(\rho_{p}^{2}\right)^{2}}{\sum_{j=1}^{m} \rho_{j}^{2}}\right]$,

(2) $\tilde{\Upsilon}_{p+m(q-1), p+m(q-1)}=E\left[\tau_{k^{\prime}} \frac{K_{m-\alpha-1}\left(\beta \sqrt{\tau_{k^{\prime}} \sum_{j=1}^{m} \rho_{j}^{2}}\right) K_{m-\alpha+1}\left(\beta \sqrt{\tau_{k^{\prime}} \sum_{j=1}^{m} \rho_{j}^{2}}\right)}{K_{m-\alpha}^{2}\left(\beta \sqrt{\tau_{k^{\prime}} \sum_{j=1}^{m} \rho_{j}^{2}}\right)} \frac{\rho_{p}^{2} \rho_{p^{\prime}}^{2}}{\sum_{j=1}^{m} \rho_{j}^{2}}\right]$,

(3) $\tilde{\Upsilon}_{p+m(p-1), p^{\prime}+m\left(p^{\prime}-1\right)}=E\left[\tau_{k^{\prime}} \frac{K_{m-\alpha-1}\left(\beta \sqrt{\tau_{k^{\prime}} \sum_{j=1}^{m} \rho_{j}^{2}}\right) K_{m-\alpha+1}\left(\beta \sqrt{\tau_{k^{\prime}} \sum_{j=1}^{m} \rho_{j}^{2}}\right)}{K_{m-\alpha}^{2}\left(\beta \sqrt{\tau_{k^{\prime}} \sum_{j=1}^{m} \rho_{j}^{2}}\right)} \frac{\rho_{p}^{2} \rho_{q}^{2}}{\sum_{j=1}^{m} \rho_{j}^{2}}\right]$.

Note that $\tilde{\Upsilon}_{p+m(q-1), p+m(q-1)}=\tilde{\Upsilon}_{p+m(p-1), p^{\prime}+m\left(p^{\prime}-1\right)}=\frac{1}{2} \tilde{\Upsilon}_{p+m(p-1), p+m(p-1)} \forall p$ 
$\forall p^{\prime} \forall q \forall q^{\prime}$. Consequently, only

$$
\varphi(\alpha, m)=\beta^{2} E\left[\tau_{k^{\prime}} \frac{K_{m-\alpha-1}\left(\beta \sqrt{\tau_{k^{\prime}} \sum_{j=1}^{m} \rho_{j}^{2}}\right) K_{m-\alpha+1}\left(\beta \sqrt{\tau_{k^{\prime}} \sum_{j=1}^{m} \rho_{j}^{2}}\right)}{K_{m-\alpha}^{2}\left(\beta \sqrt{\tau_{k^{\prime}} \sum_{j=1}^{m} \rho_{j}^{2}}\right)} \frac{\left(\rho_{p}^{2}\right)^{2}}{\sum_{j=1}^{m} \rho_{j}^{2}}\right],
$$

has to be computed and the matrix $\Upsilon$ can be written

$$
\mathbf{\Upsilon}=\frac{\varphi(\alpha, m)}{2 \beta^{4}}\left(\mathbf{M}^{T / 2} \otimes \mathbf{M}^{1 / 2}\right)\left(\mathbf{I}+\operatorname{vec}(\mathbf{I}) \operatorname{vec}^{T}(\mathbf{I})\right)\left(\mathbf{M}^{T / 2} \otimes \mathbf{M}^{1 / 2}\right) .
$$

Note that $\varphi(\alpha, m)$ is independent of $\beta$ since, $\beta^{2} \tau_{k^{\prime}} \sim \mathcal{G}(\alpha, 4)$.

\section{References}

[1] S. M. Kay, Fundamentals of Statistical Signal Processing - Detection Theory, Vol. 2, Prentice-Hall PTR, 1998.

[2] H. L. Van Trees, Detection, Estimation and Modulation Theory, Part I, John Wiley \& Sons, New York, 1968.

[3] L. L. Scharf, D. W. Lytle, Signal detection in Gaussian noise of unknown level: an invariance application, IEEE Trans.-IT 17 (1971) 404-411.

[4] S. Haykin, Array Signal Processing, Prentice-Hall Signal Processing Series, Englewood Cliffs, New Jersey, 1985.

[5] H. L. Van Trees, Detection, Estimation and Modulation Theory, Part IV: Optimum Array Processing, John Wiley \& Sons, New York, 2002.

[6] J. B. Billingsley, Ground clutter measurements for surface-sited radar, Tech. Rep. 780, MIT (February 1993).

[7] A. Farina, F. Gini, M. V. Greco, L. Verrazzani, High resolution sea clutter data: A statistical analysis of recorded live data, IEE Proceeding, Part. F 144 (3) (1997) 121-130.

[8] E. Conte, M. Longo, Characterization of radar clutter as a spherically invariant random process, IEE Proceeding, Part. F 134 (2) (1987) 191-197.

[9] K. J. Sangston, K. R. Gerlach, Coherent detection of radar targets in a nonGaussian background, IEEE Trans.-AES 30 (1994) 330-340.

[10] F. Gini, Sub-optimum coherent radar detection in a mixture of K-distributed and Gaussian clutter, IEE Proc. Radar, Sonar and Navigation 144 (1) (1997) $39-48$. 
[11] F. Gini, M. V. Greco, M. Diani, L. Verrazzani, Performance analysis of two adaptive radar detectors against non-Gaussian real sea clutter data, IEEE Trans.-AES 36 (4) (2000) 1429-1439.

[12] B. Picinbono, Spherically invariant and compound Gaussian stochastic processes, IEEE Trans.-IT (1970) 77-79.

[13] K. Yao, A representation theorem and its applications to spherically invariant random processes, IEEE Trans.-IT 19 (5) (1973) 600-608.

[14] M. Rangaswamy, D. D. Weiner, A. Ozturk, Non-Gaussian vector identification using spherically invariant random processes, IEEE Trans.-AES 29 (1) (1993) $111-124$.

[15] M. Rangaswamy, Statistical analysis of the nonhomogeneity detector for nonGaussian interference backgrounds, IEEE Trans.-SP 53 (6) (2005) 2101-2111.

[16] E. Jay, J.-P. Ovarlez, D. Declercq, P. Duvaut, Bord: Bayesian optimum radar detector, Signal Processing 83 (6) (2003) 1151-1162.

[17] F. Gini, M. V. Greco, Covariance matrix estimation for CFAR detection in correlated heavy tailed clutter, Signal Processing, special section on SP with Heavy Tailed Distributions 82 (12) (2002) 1847-1859.

[18] E. Conte, A. De Maio, G. Ricci, Recursive estimation of the covariance matrix of a compound-Gaussian process and its application to adaptive CFAR detection, IEEE Trans.-SP 50 (8) (2002) 1908-1915.

[19] S. Watts, Radar detection prediction in sea clutter using the compound Kdistribution model, IEE Proceeding, Part. F 132 (7) (1985) 613-620.

[20] T. Nohara, S. Haykin, Canada east coast trials and the K-distribution, IEE Proceeding, Part. F 138 (2) (1991) 82-88.

[21] S. Watts, Radar performance in K-distributed sea clutter, in: RTO SET Symposium on "Low Grazing Angle Clutter: Its Characterisation, Measurement and Application", RTO MP-60, 2000, pp. 372-379.

[22] I. Antipov, Statistical analysis of northern australian coastline sea clutter data, Tech. rep., Defence Science and Technology Organisation, DSTO, available at http://www.dsto.defence.gov.au/corporate/reports/DSTO-TR1236.pdf (November 2001).

[23] D. Blacknell, Comparison of parameter estimators for K-distribution, IEE Proc. Radar, Sonar and Navigation 141 (1) (1994) 45-52.

[24] W. J. J. Roberts, S. Furui, Maximum likelihood estimation of K-distribution parameters via the expectation-maximization algorithm, IEEE Trans.-SP 48 (12) (2000) 3303-3306.

[25] J. Wang, A. Dogandzic, A. Nehorai, Maximum likelihood estimation of compound-Gaussian clutter and target parameters, IEEE Trans.-SP 54 (10) (2006) 3884-3898. 
[26] D. R. Raghavan, R S, N. B. Pulsone, A generalization of the adaptive matched filter receiver for array detection in a class of a non-gaussian interference, Proc. ASAP Workshop, Lexinton, MA (1996) 499-517.

[27] N. B. Pulsone, Adaptive signal detection in non-gaussian interference, Ph.D. thesis, Northeastern University, Boston, MA (May 1997).

[28] Y. Chitour, F. Pascal, Exact maximum likelihood estimates for SIRV covariance matrix: Existence and algorithm analysis, IEEE Trans.-SP 56 (10) (2008) 45634573 .

[29] F. Gini, A radar application of a modified Cramér-Rao bound: Parameter estimation in non-Gaussian clutter, IEEE Trans.-SP 46 (7) (1998) 1945-1953.

[30] F. Pascal, P. Forster, J.-P. Ovarlez, P. Larzabal, Performance analysis of covariance matrix estimates in impulsive noise, IEEE Trans.-SP 56 (6) (2008) $2206-2217$.

[31] M. Abramowitz, I. Stegun, Handbook of Mathematical Functions, National Bureau of Standard, AMS 55, 1964.

[32] A. W. van der Vaart, Asymptotic Statistics, Cambridge University Press, 1998.

[33] K. B. Petersen, M. S. Pedersen, The matrix cookbook, available at http://matrixcookbook.com (Feb. 2008).

[34] M. Abramowitz, I. A. Stegun, Handbook of Mathematical Functions with Formulas, Graphs and Mathematical Tables, Dover Publications, inc., New York, 1972. 